JOURNAL OF THE

AMERICAN MATHEMATICAL SOCIETY

Volume 15, Number 3, Pages 531-572

S 0894-0347(02)00396-X

Article electronically published on April 5, 2002

\title{
TORIFICATION AND FACTORIZATION OF BIRATIONAL MAPS
}

\author{
DAN ABRAMOVICH, KALLE KARU, KENJI MATSUKI, \\ AND JAROSŁAW WŁODARCZYK
}

\section{CONTENTS}

0. Introduction

1. Preliminaries

2. Birational cobordisms

3. Torification 552

4. A proof of the weak factorization theorem 560

5. Generalizations 563

6. Problems related to weak factorization 5

Acknowledgements $\quad 569$

References $\quad 569$

\section{INTRODUCTION}

We work over an algebraically closed field $K$ of characteristic 0 . We denote the multiplicative group of $K$ by $K^{*}$.

0.1. Statement of the main result. The purpose of this paper is to give a proof for the following weak factorization conjecture of birational maps. We note that another proof of this theorem was given by the fourth author in [82. See section 0.13 for a brief comparison of the two approaches.

Theorem 0.1.1 (Weak Factorization). Let $\phi: X_{1} \rightarrow X_{2}$ be a birational map between complete nonsingular algebraic varieties $X_{1}$ and $X_{2}$ over an algebraically closed field $K$ of characteristic zero, and let $U \subset X_{1}$ be an open set where $\phi$ is an isomorphism. Then $\phi$ can be factored into a sequence of blowings up and blowings

Received by the editors March 14, 2000 and, in revised form, June 1, 2000.

2000 Mathematics Subject Classification. Primary 14E05.

The first author was partially supported by NSF grant DMS-9700520 and by an Alfred P. Sloan research fellowship. In addition, he would like to thank the Institut des Hautes Études Scientifiques, Centre Emile Borel (UMS 839, CNRS/UPMC), and Max Planck Institut für Mathematik for a fruitful visiting period.

The second author was partially supported by NSF grant DMS-9700520.

The third author has received no financial support from NSF or NSA during the course of this work.

The fourth author was supported in part by Polish KBN grant 2 P03 A 00516 and NSF grant DMS-0100598. 
down with nonsingular irreducible centers disjoint from $U$, namely, there exists a sequence of birational maps between complete nonsingular algebraic varieties

$$
X_{1}=V_{0} \stackrel{\varphi_{1}}{\rightarrow} V_{1} \stackrel{\varphi_{2}}{\rightarrow-} \ldots \stackrel{\varphi_{i-1}}{\rightarrow} V_{i-1} \stackrel{\varphi_{i}}{\rightarrow--} V_{i} \stackrel{\varphi_{i+1}}{\rightarrow} \cdots \stackrel{\varphi_{l-1}}{\rightarrow} V_{l-1} \stackrel{\varphi_{l}}{\rightarrow} V_{l}=X_{2}
$$

where

(1) $\phi=\varphi_{l} \circ \varphi_{l-1} \circ \cdots \varphi_{2} \circ \varphi_{1}$,

(2) $\varphi_{i}$ are isomorphisms on $U$, and

(3) either $\varphi_{i}: V_{i-1} \rightarrow V_{i}$ or $\varphi_{i}^{-1}: V_{i} \rightarrow V_{i-1}$ is a morphism obtained by blowing up a nonsingular irreducible center disjoint from $U$.

Furthermore, there is an index $i_{0}$ such that for all $i \leq i_{0}$ the map $V_{i} \rightarrow X_{1}$ is a projective morphism, and for all $i \geq i_{0}$ the map $V_{i} \rightarrow X_{2}$ is a projective morphism. In particular, if $X_{1}$ and $X_{2}$ are projective, then all the $V_{i}$ are projective.

0.2 . Strong factorization. If we insist in the assertion above that $\varphi_{1}^{-1}, \ldots, \varphi_{i_{0}}^{-1}$ and $\varphi_{i_{0}+1}, \ldots, \varphi_{l}$ be morphisms for some $i_{0}$, we obtain the following strong factorization conjecture.

Conjecture 0.2.1 (Strong Factorization). Let the situation be as in Theorem 0.1.1. Then there exists a diagram

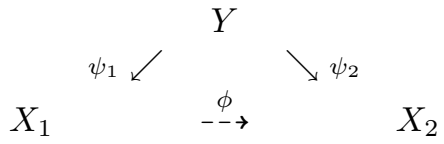

where the morphisms $\psi_{1}$ and $\psi_{2}$ are composites of blowings up of nonsingular centers disjoint from $U$.

See section 6.1 for further discussion.

0.3. Generalizations of the main theorem. We consider the following categories, in which we denote the morphisms by "broken arrows":

(1) the objects are complete nonsingular algebraic spaces over an arbitrary field $L$ of characteristic 0 , and broken arrows $X \rightarrow Y$ denote birational $L$-maps, and

(2) the objects are compact complex manifolds, and broken arrows $X \rightarrow Y$ denote bimeromorphic maps.

Given two broken arrows $\phi: X \rightarrow Y$ and $\phi^{\prime}: X^{\prime} \rightarrow Y^{\prime}$ we define an absolute isomorphism $g: \phi \rightarrow \phi^{\prime}$ as follows:

- In the case $X$ and $Y$ are algebraic spaces over $L$, and $X^{\prime}, Y^{\prime}$ are over $L^{\prime}$, then $g$ consists of an isomorphism $\sigma: \operatorname{Spec} L \rightarrow \operatorname{Spec} L^{\prime}$, together with a pair of biregular $\sigma$-isomorphisms $g_{X}: X \rightarrow X^{\prime}$ and $g_{Y}: Y \rightarrow Y^{\prime}$, such that $\phi^{\prime} \circ g_{X}=g_{Y} \circ \phi$.

- In the analytic case, $g$ simply consists of a pair of biregular isomorphisms $g_{X}: X \rightarrow X^{\prime}$ and $g_{Y}: Y \rightarrow Y^{\prime}$, such that $\phi^{\prime} \circ g_{X}=g_{Y} \circ \phi$.

Theorem 0.3.1. Let $\phi: X_{1} \rightarrow X_{2}$ be as in case (1) or (2) above. Let $U \subset X_{1}$ be an open set where $\phi$ is an isomorphism. Then $\phi$ can be factored, functorially with respect to absolute isomorphisms, into a sequence of blowings up and blowings down with nonsingular centers disjoint from $U$. Namely, to any such $\phi$ we associate a diagram in the corresponding category

$$
X_{1}=V_{0} \stackrel{\varphi_{1}}{\rightarrow} V_{1} \stackrel{\varphi_{2}}{\rightarrow--} \cdots \stackrel{\varphi_{i-1}}{\rightarrow-} V_{i-1} \stackrel{\varphi_{i}}{\stackrel{-}{\rightarrow}} V_{i} \stackrel{\varphi_{i+1}}{\rightarrow} \cdots \stackrel{\varphi_{l-1}}{\rightarrow} V_{l-1} \stackrel{\varphi_{l}}{\rightarrow} V_{l}=X_{2}
$$


where

(1) $\phi=\varphi_{l} \circ \varphi_{l-1} \circ \cdots \varphi_{2} \circ \varphi_{1}$

(2) $\varphi_{i}$ are isomorphisms on $U$, and

(3) either $\varphi_{i}: V_{i-1} \rightarrow V_{i}$ or $\varphi_{i}^{-1}: V_{i} \rightarrow V_{i-1}$ is a morphism obtained by blowing up a nonsingular center disjoint from $U$.

(4) Functoriality: if $g: \phi \rightarrow \phi^{\prime}$ is an absolute isomorphism, carrying $U$ to $U^{\prime}$, and $\varphi_{i}^{\prime}: V_{i-1}^{\prime} \rightarrow V_{i}^{\prime}$ is the factorization of $\phi^{\prime}$, then the resulting rational maps $g_{i}: V_{i} \rightarrow V_{i}^{\prime}$ give absolute isomorphisms.

(5) Moreover, there $i s$ an index $i_{0}$ such that for all $i \leq i_{0}$ the map $V_{i} \rightarrow X_{1}$ is a projective morphism, and for all $i \geq i_{0}$ the map $V_{i} \rightarrow X_{2}$ is a projective morphism.

(6) Let $E_{i} \subset V_{i}$ be the exceptional divisor of $V_{i} \rightarrow X_{1}$ (respectively, $V_{i} \rightarrow X_{2}$ ) in case $i \leq i_{0}$ (respectively, $\left.i \geq i_{0}\right)$. Then the above centers of blowing up in $V_{i}$ have normal crossings with $E_{i}$. If, moreover, $X_{1} \backslash U$ (respectively, $X_{2} \backslash U$ ) is a normal crossings divisor, then the centers of blowing up have normal crossings with the inverse images of this divisor.

Remarks. (1) Note that, in order to achieve functoriality, we cannot require the centers of blowing up to be irreducible.

(2) Functoriality implies, as immediate corollaries, the existence of factorization over any field of characteristic 0 , as well as factorization, equivariant under the action of a group $G$, of a $G$-equivariant birational map. If one assumes the axiom of choice, then a standard argument shows that equivariance implies functoriality. In our proofs we do not use the axiom of choice, with the exceptions of (1) existence of an algebraic closure, and (2) section 5.6, where showing functoriality without the assumption of the axiom of choice would require revising some of the arguments of 56 . We hope that the interested reader will be able to rework our arguments without the assumption of the axiom of choice if this becomes desirable.

(3) The same theorem holds true for varieties or algebraic spaces of dimension $d$ over a perfect field of characteristic $p>0$ assuming that canonical embedded resolution of singularities holds true for varieties or algebraic spaces of dimension $d+1$ in characteristic $p$. The proof for varieties goes through word for word as in this paper, while for the algebraic space case one needs to recast some of our steps from the Zariski topology to the étale topology (see [38], [53]).

(4) While this theorem clearly implies the main theorem as a special case, we prefer to carry out the proof of the main theorem throughout the text, and to indicate the changes one needs to perform for proving Theorem 0.3 .1 in section 5

0.4. Applying the theorem. Suppose one is given a biregular invariant of nonsingular projective varieties and one is interested in the behavior of this invariant under birational transformations. Traditionally, one would (1) study the behavior of the invariant under blowings up with nonsingular centers, (2) form a conjecture according to this study, and finally (3) attempt to prove the conjecture using additional ideas.

Sometimes such additional ideas turn out to be fairly simple (e.g. birational invariance of spaces of differential forms). Sometimes they use known but deep results (e.g. Hodge theory for showing the birational invariance of $H^{i}\left(X, \mathcal{O}_{X}\right)$ in 
characteristic 0 ; abelian varieties for the birational invariance of $H^{1}\left(X, \mathcal{O}_{X}\right)$ in general; or Deligne's work on the Weil conjectures for the results of [47]). Sometimes they lead to the development of beautiful new theories (e.g. Motivic integration for the invariance of Hodge numbers of birational Calabi-Yau varieties, [45], 7], [8], [22], [50; see also [10] where our theorem is applied).

Our theorem implies that, in characteristic 0, step (3) above is no longer necessary: once such a conjecture is compatible with blowings up with nonsingular centers, it holds for any birational map. At the time of the revision of this paper we know of two announced applications for which no alternative methods of proof are known: (a) construction of elliptic genera of singular varieties by L. Borisov and A. Libgober [11, and (b) showing that the algebraic cobordism ring of a field is the Lazard ring, by M. Levine and F. Morel (48, Théorème 1.1, 49]).

When we set out to write this paper, we attempted to give a statement detailed enough and general enough to apply in all applications we had imagined. As soon as the paper was circulated, it became clear that there are applications not covered by Theorem 0.3.1, even though the methods apply. In the preprint [27] of H. Gillet and Ch. Soulé, the authors use the behavior of localized Todd classes under proper birational maps of schemes which are projective over a discrete valuation ring of residue characteristic 0 . In their proof they rely on deep (and yet unpublished in complete form) results of J. Franke [25]; alternatively, they could have used weak factorization for such maps. While proving this case may be a straightforward exercise using our methods, this would still leave a plethora of other possible applications (more general base schemes, real analytic geometry, $p$-adic analytic geometry, to name a few).

One could imagine a statement of a general "weak factorization - type" result relying on a minimal set of axioms needed to carry out our line of proof of weak factorization. We decided to spare ourselves and the reader such formalism in this paper.

0.5. Early origins of the problem. The history of the factorization problem of birational maps could be traced back to the Italian school of algebraic geometers, who already knew that the operation of blowing up points on surfaces is a fundamental source of richness for surface geometry: the importance of the strong factorization theorem in dimension 2 (see [83]) cannot be overestimated in the analysis of the birational geometry of algebraic surfaces. We can only guess that Zariski, possibly even members of the Italian school, contemplated the problem in higher dimension early on, but refrained from stating it before results on resolution of singularities were available. The question of strong factorization was explicitly stated by Hironaka as "Question $\left(\mathrm{F}^{\prime}\right)$ " in [30], Chapter $0, \S 6$, and the question of weak factorization was raised in 61. The problem remained largely open in higher dimensions despite the efforts and interesting results of many (see e.g. Crauder [15], Kulikov [46], Moishezon [55], Schaps [72], Teicher [76]). Many of these were summarized by Pinkham [64, where the weak factorization conjecture is explicitly stated.

0.6. The toric case. For toric birational maps, the equivariant versions of the weak and strong factorization conjectures were posed in [61] and came to be known as Oda's weak and strong conjectures. While the toric version can be viewed as a special case of the general factorization conjectures, many of the examples demonstrating the difficulties in higher dimensions are in fact toric (see Hironaka [29], 
Sally [70, Shannon [73]). Thus Oda's conjecture presented a substantial challenge and combinatorial difficulty. In dimension 3, Danilov's proof of Oda's weak conjecture 21] was later supplemented by Ewald [24]. Oda's weak conjecture was solved in arbitrary dimension by J. Włodarczyk in [80], and another proof was given by R. Morelli in 56 (see also [57, and 4, where the result is generalized to the toroidal situation). An important combinatorial notion which Morelli introduced into this study is that of a cobordism between fans. The algebro-geometric realization of Morelli's combinatorial cobordism is the notion of a birational cobordism introduced in 81 .

Our proof of the main theorem relies on toric weak factorization. This remains as one of the most difficult theorems leading to our result.

In [56], R. Morelli also proposed a proof of Oda's strong conjecture. A gap in this proof, which was not noticed in 4], was recently discovered by K. Karu. As far as we know, Oda's strong conjecture stands unproven at present even in dimension 3 .

0.7. A local version. There is a local version of the factorization conjecture, formulated and proved in dimension 2 by Abhyankar (1], Theorem 3). Christensen [13. posed the problem in general and solved it for some special cases in dimension 3. Here the varieties $X_{1}$ and $X_{2}$ are replaced by appropriate birational local rings dominated by a fixed valuation, and blowings up are replaced by monoidal transforms subordinate to the valuation. The weak form of this local conjecture, as well as the strong version in the threefold case, was recently solved by S. D. Cutkosky in a series of papers [16, 17]. Cutkosky also shows that the strong version of the conjecture follows from Oda's strong factorization conjecture for toric morphisms. In a sense, Cutkosky's result says that the only local obstructions to solving the global strong factorization conjecture lie in the toric case.

0.8. Birational cobordisms. Our method is based upon the theory of birational cobordisms 81]. As mentioned above, this theory was inspired by the combinatorial notion of polyhedral cobordisms of R. Morelli [56], which was used in his proof of weak factorization for toric birational maps.

Given a birational map $\phi: X_{1} \rightarrow X_{2}$, a birational cobordism $B_{\phi}\left(X_{1}, X_{2}\right)$ is a variety of dimension $\operatorname{dim}\left(X_{1}\right)+1$ with an action of the multiplicative group $K^{*}$. It is analogous to the usual cobordism $B\left(M_{1}, M_{2}\right)$ between differentiable manifolds $M_{1}$ and $M_{2}$ given by a Morse function $f$ (and in fact in the Kähler case the momentum map of $\mathbb{C}^{*}$ is a Morse function, making the analogy more direct). In the differential setting one can construct an action of the additive real group $\mathbb{R}$, where the "time" $t \in \mathbb{R}$ acts as a diffeomorphism induced by integrating the vector field $\operatorname{grad}(f)$; hence the multiplicative group $\left(\mathbb{R}_{>0}, \times\right)=\exp (\mathbb{R},+)$ acts as well. The critical points of $f$ are precisely the fixed points of the action of the multiplicative group, and the homotopy type of fibers of $f$ changes when we pass through these critical points (see [54]). Analogously, in the algebraic setting "passing through" the fixed points of the $K^{*}$-action induces a birational transformation. Looking at the action on the tangent space at each fixed point, we obtain a locally toric description of the transformation. This already gives the main result of [81]: a factorization of $\phi$ into certain locally toric birational transformations among varieties with locally toric structures. More precisely, it is shown in [81] that the intermediate varieties have abelian quotient singularities, and the locally toric birational transformations can be factored in terms of weighted blowings up. Such birational transformations 
can also be interpreted using the work of Brion-Procesi, Thaddeus, Dolgachev-Hu and others (see [12, 77, 78, 23]), which describes the change of Geometric Invariant Theory quotients associated to a change of linearization. We use such methods in section 2.5] in showing that the intermediate varieties are projective over $X_{1}$ or $X_{2}$. A variant of our construction using Geometric Invariant Theory, in terms of Thaddeus's "Master Space", is given by Hu and Keel in 34.

0.9. Locally toric versus toroidal structures. Considering the fact that weak factorization has been proven for toroidal birational maps ([80, [56], 4]), one might naïvely think that a locally toric factorization, as indicated in the previous paragraph, would already provide a proof for Theorem 0.1 .1

However, in the locally toric structure obtained from a cobordism, the embedded tori chosen may vary from point to point, while a toroidal structure (see Definition 1.5.1) requires the embedded tori to be induced from one fixed open set. Thus there is still a gap between the notion of locally toric birational transformations and that of toroidal birational maps. Developing a method for bridging over this gap is the main contribution of this paper.

0.10. Torification. In order to bridge over this gap, we follow ideas introduced by Abramovich and de Jong in [2], and blow up suitable open subsets, called quasielementary cobordisms, of the birational cobordism $B_{\phi}\left(X_{1}, X_{2}\right)$ along torific ideals. This operation induces a toroidal structure in a neighborhood of each connected component $F$ of the fixed point set, on which the action of $K^{*}$ is a toroidal action (we say that the blowing up torifies the action of $K^{*}$ ). Now the birational transformation "passing through $F$ " is toroidal. We use canonical resolution of singularities to desingularize the resulting varieties, bringing ourselves to a situation where we can apply the factorization theorem for toroidal birational maps. This completes the proof of Theorem 0.1 .1 .

0.11. Relation with the minimal model program. It is worthwhile to note the relation of the factorization problem to the development of Mori's program. Hironaka [28] used the cone of effective curves to study the properties of birational morphisms. This direction was further developed and given a decisive impact by Mori 58, who introduced the notion of extremal rays and systematically used it in an attempt to construct minimal models in higher dimension, called the minimal model program. Danilov [21] introduced the notion of canonical and terminal singularities in conjunction with the toric factorization problem. This was developed by Reid into a general theory of these singularities [66, 67, which appear in an essential way in the minimal model program. The minimal model program is so far proven up to dimension 3 ([59], see also [39, 40, 41, 44, 74]), and for toric varieties in arbitrary dimension (see [68]). In the steps of the minimal model program one is only allowed to contract a divisor into a variety with terminal singularities, or to perform a flip, modifying some codimension $\geq 2$ loci. This allows a factorization of a given birational morphism into such "elementary operations". An algorithm to factor birational maps among uniruled varieties, known as Sarkisov's program, has been developed and carried out in dimension 3 (see [71, 69, 14], and see [52] for the toric case in arbitrary dimension). Still, we do not know of a way to solve the classical factorization problem using such a factorization. 
0.12. Relation with the toroidalization problem. In 3], Theorem 2.1, it is proven that given a morphism of projective varieties $X \rightarrow B$, there are modifications $m_{X}: X^{\prime} \rightarrow X$ and $m_{B}: B^{\prime} \rightarrow B$, with a lifting $X^{\prime} \rightarrow B^{\prime}$ which has a toroidal structure. The toroidalization problem (see [3], [4], [43]) is that of obtaining such $m_{X}$ and $m_{B}$ which are composites of blowings up with nonsingular centers (maybe even with centers supported only over the locus where $X \rightarrow B$ is not toroidal).

The proof in [3] relies on the work of de Jong [36] and methods of [2]. The authors of the present paper have tried to use these methods to approach the factorization conjectures, so far without success; one notion we do use in this paper is the torific ideal of [2]. It would be interesting if one could turn this approach on its head and prove a result on toroidalization using factorization.

More on this in section 6.2.

0.13. Relation with the proof in 82 . Another proof of the weak factorization theorem was given independently by the fourth author in 82 . The main difference between the two approaches is the following: in the current paper we are using objects such as torific ideals defined locally on each quasi-elementary piece of a cobordism. The blowing up of a torific ideal gives the quasi-elementary cobordism a toroidal structure. These toroidal modifications are then pieced together using canonical resolution of singularities. In contrast, in 82 one works globally: a new combinatorial theory of stratified toroidal varieties and appropriate morphisms between them is developed, which allows one to apply Morelli's $\pi$-desingularization algorithm directly to the entire birational cobordism. This stratified toroidal variety structure on the cobordism is somewhere in between our notions of locally toric and toroidal structures.

0.14. Outline of the paper. In section $\square$ we discuss locally toric and toroidal structures. We also use elimination of indeterminacies of a rational map to reduce the proof of Theorem 0.1.1 to the case where $\phi$ is a projective birational morphism.

Suppose now we have a projective birational morphism $\phi: X_{1} \rightarrow X_{2}$. In section 2 we apply the theory of birational cobordisms to obtain a slightly refined version of factorization into locally toric birational maps, first proven in [81]. Our cobordism $B$ is relatively projective over $X_{2}$, and using a geometric invariant theory analysis, inspired by Thaddeus's work, we show that the intermediate varieties can be chosen to be projective over $X_{2}$.

In section 3 we utilize a factorization of the cobordism $B$ into quasi-elementary pieces $B_{a_{i}}$, and for each piece construct an ideal sheaf $I$ (Definition 3.1.4) whose blowing up torifies the action of $K^{*}$ on $B_{a_{i}}$ (Proposition 3.2.5). In other words, $K^{*}$ acts toroidally on the variety obtained by blowing up $B_{a_{i}}$ along $I$.

In section 4 we prove the weak factorization theorem by putting together the toroidal birational maps obtained from the torification of the quasi-elementary cobordisms (Proposition 4.2.1), and applying toroidal weak factorization. The main tool in this step is canonical resolution of singularities.

In section 5 we prove Theorem 0.3 .1 We then discuss some problems related to strong factorization in section [6.

\section{Preliminaries}

1.1. Quotients. We use the following definitions for quotients. Suppose a reductive group $G$ acts on an algebraic variety $X$. We denote by $X / G$ the space of orbits, 
and by $X / / G$ the space of equivalence classes of orbits, where the equivalence relation is generated by the condition that two orbits are equivalent if their closures intersect; such a space is endowed with a scheme structure which satisfies the usual universal property, if such a structure exists. In such a case, the space $X / / G$ is called a categorical quotient and the space $X / G$ is called a geometric quotient.

A special case where $X / / G$ exists as a scheme is the following: suppose there is an affine $G$-invariant morphism $\pi: X \rightarrow Y$. Then we have $X / / G=\operatorname{Spec}_{Y}\left(\left(\pi_{*} \mathcal{O}_{X}\right)^{G}\right)$. When this condition holds we say that the action of $G$ on $X$ is relatively affine.

A particular case of this occurs in geometric invariant theory (discussed in section 2.5), where the action of $G$ on the open set of points which are semistable with respect to a fixed linearization is relatively affine.

1.2. Canonical resolution of singularities and canonical principalization. In the following (especially Lemma 1.3.1] section 4.2, section [5), we will use canonical versions of Hironaka's theorems on resolution of singularities and principalization of an ideal, proved in [9, 79].

1.2.1. Canonical resolution. Following Hironaka, by a canonical embedded resolution of singularities $\widetilde{W} \rightarrow W$ we mean a desingularization procedure uniquely associating to $W$ a composite of blowings up with nonsingular centers, satisfying a number of conditions. In particular:

(1) "Embedded" means the following: assume the sequence of blowings up is applied when $W \subset U$ is a closed embedding with $U$ nonsingular. Denote by $E_{i}$ the exceptional divisor at some stage of the blowing up. Then (a) $E_{i}$ is a normal crossings divisor, and has normal crossings with the center of blowing up, and (b) at the last stage $\widetilde{W}$ has normal crossings with $E_{i}$.

(2) "Canonical" means "functorial with respect to smooth morphisms and field extensions", namely, if $\theta: V \rightarrow W$ is either a smooth morphism or a field extension, then the formation of the ideals blown up commutes with pulling back by $\theta$; hence $\theta$ can be lifted to a smooth morphism $\widetilde{\theta}: \widetilde{V} \rightarrow \widetilde{W}$.

In particular: (a) if $\theta: W \rightarrow W$ is an automorphism (of schemes, not necessarily over $K$ ), then it can be lifted to an automorphism $\widetilde{W} \rightarrow \widetilde{W}$, and (b) the canonical resolution behaves well with respect to étale morphisms: if $V \rightarrow W$ is étale, we get an étale morphism of canonical resolutions $\widetilde{V} \rightarrow \widetilde{W}$.

An important consequence of these conditions is that all the centers of blowing up lie over the singular locus of $W$.

We note that the resolution processes in the work of Bierstone and Milman and of Villamayor commute with arbitrary formally smooth morphisms (in particular smooth morphisms, field extensions, and formal completions), though the treatment in any of the published works does not seem to state that explicitly.

1.2.2. Compatibility with a normal crossings divisor. If $W \subset U$ is embedded in a nonsingular variety, and $D \subset U$ is a normal crossings divisor, then a variant of the resolution procedure allows one to choose the centers of blowing up to have normal crossings with $D_{i}+E_{i}$, where $D_{i}$ is the inverse image of $D$. This follows since the resolution setup, as in [9], allows including such a divisor in "year 0".

1.2.3. Principalization. By canonical principalization of an ideal sheaf in a nonsingular variety we mean "the canonical embedded resolution of singularities of the subscheme defined by the ideal sheaf making it a divisor with normal crossings"; 
i.e., a composite of blowings up with nonsingular centers such that the total transform of the ideal is a divisor with simple normal crossings. Canonical embedded resolution of singularities of an arbitrary subscheme, not necessarily reduced or irreducible, is discussed in section 11 of [9], and this implies canonical principalization, as one simply needs to blow up $\widetilde{W}$ at the last step.

1.2.4. Elimination of indeterminacies. Now let $\phi: W_{1} \rightarrow W_{2}$ be a birational map and $U \subset W_{1}$ an open set on which $\phi$ restricts to a morphism. By elimination of indeterminacies of $\phi$ we mean a morphism $e: W_{1}^{\prime} \rightarrow W_{1}$, obtained by a sequence of blowings up with nonsingular centers disjoint from $U$, such that the birational map $\phi \circ e$ is a morphism.

Elimination of indeterminacies can be reduced to principalization of an ideal sheaf: if one is given an ideal sheaf $I$ on $W_{1}$ with blowing up $W_{1}^{\prime \prime}=B l_{I}\left(W_{1}\right)$ such that the birational map $W_{1}^{\prime \prime} \rightarrow W_{2}$ is a morphism, and if $W_{1}^{\prime} \rightarrow W_{1}$ is the result of principalization of $I$, then the birational map $W_{1}^{\prime} \rightarrow W_{1}^{\prime \prime}$ is a morphism, therefore the same is true for $W_{1}^{\prime} \rightarrow W_{2}$. If the support of the ideal $I$ is disjoint from the open set $U$ where $\phi$ is an morphism, then the centers of blowing up giving $W_{1}^{\prime} \rightarrow W_{1}$ are disjoint from $U$.

Proving that such an ideal $I$ exists (say, in the nonprojective case), and in a sufficiently natural manner for proving functoriality (even if $W_{i}$ are projective), is nontrivial. We make use of Hironaka's version of Chow's lemma, as follows.

We may assume that $\phi^{-1}$ is a morphism; otherwise we replace $W_{2}$ by the closure of the graph of $\phi$. Now we use Chow's lemma, proven by Hironaka in general in 31], Corollary 2, p. 504, as a consequence of his flattening procedure: there exists an ideal sheaf $I$ on $W_{1}$ such that the blowing up of $W_{1}$ along $I$ factors through $W_{2}$. Hence the canonical principalization of $I$ also factors through $W_{2}$.

Although it is not explicitly stated by Hironaka, the ideal $I$ is the unit ideal in the complement of the open set $U$ : the blowing up of $I$ consists of a sequence of permissible blowings up (31], Definition 4.4.3, p. 537), each of which is supported in the complement of $U$. Another important fact is that the ideal $I$ is invariant, namely, it is functorial under absolute isomorphisms: if $\phi^{\prime}: W_{1}^{\prime} \rightarrow W_{2}^{\prime}$ is another proper birational map, with corresponding ideal $I^{\prime}$, and $\theta_{i}: W_{i} \rightarrow W_{i}^{\prime}$ are isomorphisms such that $\phi^{\prime} \circ \theta_{1}=\theta_{2} \circ \phi$, then $\theta_{1}^{*} I^{\prime}=I$. This follows simply because at no point in Hironaka's flattening procedure is there a need for any choice.

It must be pointed out that Hironaka's flattening procedure, and therefore the choice of the ideal $I$, does not commute with smooth morphisms in general - in fact Hironaka gives an example where it does not commute with localization.

The same results hold for analytic and algebraic spaces. While Hironaka states his result only in the analytic setting, the arguments hold in the algebraic setting as well. See [65] for an earlier treatment of the case of varieties.

We emphasize again that Chow's lemma in the analytic setting, and its delicate properties in both the algebraic and analytic settings, rely on Hironaka's difficult flattening theorem (see [31, or the algebraic counterpart 65]).

1.3. Reduction to projective morphisms. We start with a birational map

$$
\phi: X_{1} \rightarrow X_{2}
$$

between complete nonsingular algebraic varieties $X_{1}$ and $X_{2}$ defined over $K$ and restricting to an isomorphism on an open set $U$. 
Lemma 1.3.1 (Hironaka). There is a commutative diagram

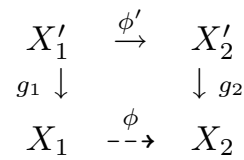

such that $g_{1}$ and $g_{2}$ are composites of blowings up with nonsingular centers disjoint from $U$, and $\phi^{\prime}$ is a projective birational morphism.

Proof. By Hironaka's theorem on elimination of indeterminacies (see 1.2.4 above), there is a morphism $g_{2}: X_{2}^{\prime} \rightarrow X_{2}$ which is a composite of blowings up with nonsingular centers disjoint from $U$, such that the birational map $h:=\phi^{-1} \circ g_{2}$ : $X_{2}^{\prime} \rightarrow X_{1}$ is a morphism:

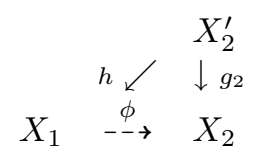

By the same theorem, there is a morphism $g_{1}: X_{1}^{\prime} \rightarrow X_{1}$ which is a composite of blowings up with nonsingular centers disjoint from $U$, such that $\phi^{\prime}:=h^{-1} \circ g_{1}$ : $X_{1}^{\prime} \rightarrow X_{2}^{\prime}$ is a morphism. Since the composite $h \circ \phi^{\prime}=g_{1}$ is projective, it follows that $\phi^{\prime}$ is projective.

Thus we may replace $X_{1} \rightarrow X_{2}$ by $X_{1}^{\prime} \rightarrow X_{2}^{\prime}$ and assume from now on that $\phi$ is a projective morphism.

Note that, by the properties of canonical principalization and Hironaka's flattening, the formation of $\phi^{\prime}: X_{1}^{\prime} \rightarrow X_{2}^{\prime}$ is functorial under absolute isomorphisms, and the blowings up have normal crossings with the appropriate divisors. This will be used in the proof of Theorem 0.3 .1 (see section 5).

1.4. Toric varieties. Let $N \cong \mathbb{Z}^{n}$ be a lattice and $\sigma \subset N_{\mathbb{R}}$ a strictly convex rational polyhedral cone. We denote the dual lattice by $M$ and the dual cone by $\sigma^{\vee} \subset M_{\mathbb{R}}$. The affine toric variety $X=X(N, \sigma)$ is defined as

$$
X=\operatorname{Spec} K\left[M \cap \sigma^{\vee}\right] \text {. }
$$

For $m \in M \cap \sigma^{\vee}$ we denote its image in the semigroup algebra $K\left[M \cap \sigma^{\vee}\right]$ by $z^{m}$.

More generally, the toric variety corresponding to a fan $\Sigma$ in $N_{\mathbb{R}}$ is denoted by $X(N, \Sigma)$; see [26], 62].

If $X_{1}=X\left(N, \Sigma_{1}\right)$ and $X_{2}=X\left(N, \Sigma_{2}\right)$ are two toric varieties, the embeddings of the torus $T=\operatorname{Spec} K[M]$ in both of them define a toric (i.e., $T$-equivariant) birational map $X_{1} \rightarrow X_{2}$.

Suppose $K^{*}$ acts effectively on an affine toric variety $X=X(N, \sigma)$ as a oneparameter subgroup of the torus $T$, corresponding to a primitive lattice point $a \in N$. If $t \in K^{*}$ and $m \in M$, the action on the monomial $z^{m}$ is given by

$$
t^{*}\left(z^{m}\right)=t^{(a, m)} \cdot z^{m}
$$

where $(\cdot, \cdot)$ is the natural pairing on $N \times M$. The $K^{*}$-invariant monomials correspond to the lattice points $M \cap a^{\perp}$, hence

$$
X / / K^{*} \cong \operatorname{Spec} K\left[M \cap \sigma^{\vee} \cap a^{\perp}\right] .
$$

If $a \notin \pm \sigma$, then $\sigma^{\vee} \cap a^{\perp}$ is a full-dimensional cone in $a^{\perp}$, and it follows that $X / / K^{*}$ is again an affine toric variety, defined by the lattice $\pi(N)$ and cone $\pi(\sigma)$, where 
$\pi: N_{\mathbb{R}} \rightarrow N_{\mathbb{R}} / \mathbb{R} \cdot a$ is the projection. This quotient is a geometric quotient precisely when $\pi: \sigma \rightarrow \pi(\sigma)$ is a bijection.

1.5. Locally toric and toroidal structures. There is some confusion in the literature between the notion of toroidal embeddings and toroidal morphisms (42, [3]) and that of toroidal varieties (see [20]), which we prefer to call locally toric varieties. A crucial issue in this paper is the distinction between the two notions.

Definition 1.5.1. (1) A variety $W$ is locally toric if for every closed point $p \in W$ there exists an open neighborhood $V_{p} \subset W$ of $p$ and an étale morphism $\eta_{p}: V_{p} \rightarrow X_{p}$ to a toric variety $X_{p}$. Such a morphism $\eta_{p}$ is called a toric chart at $p$.

(2) An open embedding $U \subset W$ is a toroidal embedding if for every closed point $p \in W$ there exists a toric chart $\eta_{p}: V_{p} \rightarrow X_{p}$ at $p$ such that $U \cap V_{p}=\eta_{p}^{-1}(T)$, where $T \subset X_{p}$ is the torus. We call such charts toroidal. Sometimes we omit the open set $U$ from the notation and simply say that a variety is toroidal.

(3) We say that a locally toric (respectively, toroidal) chart on a variety is compatible with a divisor $D \subset W$ if $\eta_{p}^{-1}(T) \cap D=\emptyset$, i.e., $D$ corresponds to a toric divisor on $X_{p}$.

A toroidal embedding $U \subset X$ can equivalently be specified by the pair $\left(X, D_{X}\right)$, where $D_{X}$ is the reduced Weil divisor supported on $X \backslash U$. We will sometimes interchange between $U \subset X$ and $\left(X, D_{X}\right)$ for denoting a toroidal structure on $X$. A divisor $D^{\prime}$ is compatible with the toroidal structure $\left(X, D_{X}\right)$ if it is supported in $D_{X}$.

For example, the affine line $\mathbb{A}^{1}$ is clearly locally toric, $\mathbb{A}^{1} \backslash\{0\} \subset \mathbb{A}^{1}$ is a toroidal embedding, and $\mathbb{A}^{1} \subset \mathbb{A}^{1}$ is a different toroidal embedding, where a chart at the point 0 can be obtained by translation from the point 1 .

Toroidal embeddings can be naturally made into a category:

Definition 1.5.2. Let $U_{i} \subset W_{i}(i=1,2)$ be toroidal embeddings. A proper birational morphism $f: W_{1} \rightarrow W_{2}$ is said to be toroidal if, for every closed point $q \in W_{2}$ and any $p \in f^{-1} q$, there is a diagram of fiber squares

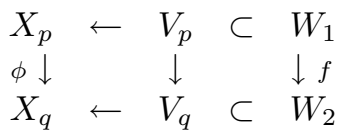

where

- $\eta_{p}: V_{p} \rightarrow X_{p}$ is a toroidal chart at $p$,

- $\eta_{q}: V_{q} \rightarrow X_{q}$ is a toroidal chart at $q$, and

- $\phi: X_{p} \rightarrow X_{q}$ is a toric morphism.

Remarks. (1) A toroidal embedding as defined above is a toroidal embedding without self-intersection according to the definition in [42], and a birational toroidal morphism satisfies the condition of allowability in [42].

(2) To a toroidal embedding $\left(U_{W} \subset W\right)$ one can associate a polyhedral complex $\Delta_{W}$, such that proper birational toroidal morphisms to $W$, up to isomorphisms, are in one-to-one correspondence with certain subdivisions of the complex (see [42]). It follows from this that the composition of two proper birational toroidal morphisms $W_{1} \rightarrow W_{2}$ and $W_{2} \rightarrow W_{3}$ is again toroidal: the first morphism corresponds to a subdivision of $\Delta_{W_{2}}$, the second one to a 
subdivision of $\Delta_{W_{3}}$, hence their composition is the unique toroidal morphism corresponding to the subdivision $\Delta_{W_{1}}$ of $\Delta_{W_{3}}$.

(3) Some of the many issues surrounding these definitions we avoided discussing here are addressed in the third author's lecture notes [53].

We now turn to birational maps:

Definition 1.5.3 (30], 35]). Let $\psi: W_{1} \rightarrow W_{2}$ be a rational map defined on a dense open subset $U$. Denote by $\Gamma_{\psi}$ the closure of the graph of $\psi_{U}$ in $W_{1} \times W_{2}$. We say that $\psi$ is proper if the projections $\Gamma_{\psi} \rightarrow W_{1}$ and $\Gamma_{\psi} \rightarrow W_{2}$ are both proper.

Definition 1.5.4. Let $U_{i} \subset W_{i}$ be toroidal embeddings. A proper birational map $\psi: W_{1} \rightarrow W_{2}$ is said to be toroidal if there exists a toroidal embedding $U_{Z} \subset Z$ and a commutative diagram

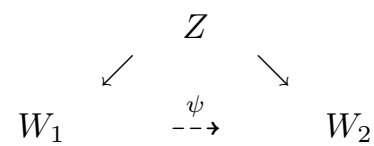

where $Z \rightarrow W_{i}(i=1,2)$ are proper birational toroidal morphisms. In particular, a proper birational toroidal map induces an isomorphism between the open sets $U_{1}$ and $U_{2}$.

Remarks. (1) It follows from the correspondence between proper birational toroidal morphisms and subdivisions of polyhedral complexes that the composition of toroidal birational maps given by $W_{1} \leftarrow Z_{1} \rightarrow W_{2}$ and $W_{2} \leftarrow$ $Z_{2} \rightarrow W_{3}$ is again toroidal. Indeed, if $Z_{1} \rightarrow W_{2}$ and $Z_{2} \rightarrow W_{2}$ correspond to two subdivisions of $\Delta_{W_{2}}$, then a common refinement of the two subdivisions corresponds to a toroidal embedding $Z$ such that $Z \rightarrow Z_{1}$ and $Z \rightarrow Z_{2}$ are toroidal morphisms. For example, the coarsest refinement corresponds to taking for $Z$ the normalization of the closure of the graph of the birational map $Z_{1} \rightarrow Z_{2}$. The composite maps $Z \rightarrow W_{i}$ are all toroidal birational morphisms.

(2) It can be shown that a morphism between toroidal embeddings which is a toroidal birational map in the sense of Definition 1.5.4 is a toroidal morphism in the sense of Definition 1.5.2 In other words, Definitions 1.5.2 and 1.5.4 are compatible.

For locally toric varieties, there are no satisfactory analogues of the definitions of toroidal morphisms and birational maps. One can define a "locally toric morphism" to be one which is toric on suitable toric charts, but this notion is neither stable under composition nor amenable to combinatorial manipulations. An extensive and quite delicate theory involving stratifications of locally toric varieties is developed in [82] in order to resolve this issue. Here we use a different remedy. We define a restrictive class of birational transformations between locally toric and toroidal varieties, in which all charts are "uniform" over a common base $Y$. These are still not stable under composition, but their local combinatorial nature suffices for our goals. These are the only transformations we will need in the considerations of the current paper. 
Definition 1.5.5. (1) A tightly locally toric birational transformation is a proper birational map $\psi: W_{1} \rightarrow W_{2}$ together with a diagram of birational maps

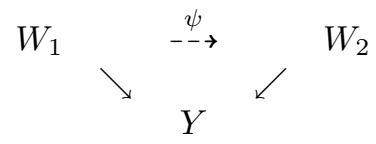

between locally toric varieties $W_{1}$ and $W_{2}$ satisfying the following condition:

For every closed point $q \in Y$ there exist a toric chart $\eta_{q}: V_{q} \rightarrow X_{q}$ at $q$, and a diagram of fibered squares

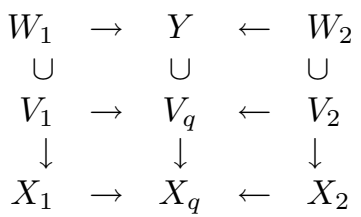

such that

(a) $V_{i} \rightarrow X_{i}$ are toric charts for $W_{i}, i=1,2$, and

(b) $X_{i} \rightarrow X_{q}$ are toric morphisms

(2) Analogously, let $U_{i} \subset W_{i}$ be toroidal embeddings. A tightly toroidal birational transformation between them is a tightly locally toric birational transformation $\psi: W_{1} \rightarrow W_{2}$ where the toric charts above can be chosen to be toroidal.

Remark. While tightly locally toric birational transformations are essential in our arguments, tightly toroidal transformations are not: the argument used before to show that a composition of toroidal birational maps is toroidal shows that a tightly toroidal birational transformation gives a toroidal birational map. This is the only property of such transformations we will use.

1.6. Weak factorization for toroidal birational maps. The weak factorization theorem for proper birational toric maps can be extended to the case of proper birational toroidal maps. This is proved in [4 for toroidal morphisms, using the correspondence between birational toroidal morphisms and subdivisions of polyhedral complexes. The general case of a toroidal birational map $W_{1} \leftarrow Z \rightarrow W_{2}$ can be deduced from this, as follows. By toroidal resolution of singularities we may assume $Z$ is nonsingular. We apply toroidal weak factorization to the morphisms $Z \rightarrow W_{i}$, to get a sequence of toroidal birational maps

$W_{1}=V_{1} \rightarrow V_{2} \rightarrow \rightarrow \cdots \rightarrow V_{l-1} \rightarrow V_{l}=Z \rightarrow V_{l+1} \rightarrow \rightarrow \cdots \rightarrow V_{k-1} \rightarrow V_{k}=W_{2}$

consisting of toroidal blowings up and down with nonsingular centers.

We state this result for later reference:

Theorem 1.6.1. Let $U_{1} \subset W_{1}$ and $U_{2} \subset W_{2}$ be nonsingular toroidal embeddings. Let $\psi: W_{1} \rightarrow W_{2}$ be a proper toroidal birational map. Then $\phi$ can be factored into a sequence of toroidal birational maps consisting of toroidal blowings up and down of nonsingular centers in nonsingular toroidal embeddings.

This does not immediately imply that one can choose a factorization satisfying a projectivity statement as in the main theorem, or in a functorial manner. We will show these facts in sections 2.7 and 5 respectively. It should be mentioned that if toric strong factorization is true, then the toroidal case follows. 


\subsection{Locally toric and toroidal actions.}

Definition 1.7.1 (see 60, p. 198). Let $V$ and $X$ be varieties with relatively affine $K^{*}$-actions, and let $\eta: V \rightarrow X$ be a $K^{*}$-equivariant étale morphism. Then $\eta$ is said to be strongly étale if

(i) the quotient map $V / / K^{*} \rightarrow X / / K^{*}$ is étale, and

(ii) the natural map

$$
V \rightarrow X \underset{X / / K^{*}}{\times} V / / K^{*}
$$

is an isomorphism.

Definition 1.7.2. (1) Let $W$ be a locally toric variety with a $K^{*}$-action, such that $W / / K^{*}$ exists. We say that the action is locally toric if for any closed point $p \in W$ we have a toric chart $\eta_{p}: V_{p} \rightarrow X_{p}$ at $p$ and a one-parameter subgroup $K^{*} \subset T_{p}$ of the torus in $X_{p}$, satisfying

- $V_{p}=\pi^{-1} \pi V_{p}$, where $\pi: W \rightarrow W / / K^{*}$ is the projection;

- $\eta_{p}$ is $K^{*}$-equivariant and strongly étale.

(2) If $U \subset W$ is a toroidal embedding, we say that $K^{*}$ acts toroidally on $W$ if the charts above can be chosen toroidal.

The definition above is equivalent to the existence of the following diagram of fiber squares:

$$
\begin{array}{ccccc}
X_{p} & \leftarrow & V_{p} & \subset & W \\
\downarrow & & \downarrow & & \downarrow f \\
X / / K^{*} & \leftarrow & V_{p} / / K^{*} & \subset & W / / K^{*}
\end{array}
$$

where the horizontal maps provide toric (resp. toroidal) charts in $W$ and $W / / K^{*}$. It follows that the quotient of a locally toric variety by a locally toric action is again locally toric; the same holds in the toroidal case.

Remark. If we do not insist on the charts being strongly étale, then the morphism of quotients may fail to be étale. Consider, for instance, the space $X=$ Spec $K\left[x, x^{-1}, y\right]$ with the action $t(x, y)=\left(t^{2} x, t^{-1} y\right)$. The quotient is $X / K^{*}=$ Spec $K\left[x y^{2}\right]$. There is an equivariant étale cover $V=\operatorname{Spec} K\left[u, u^{-1}, y\right]$ with the action $t(u, y)=\left(t u, t^{-1} y\right)$, where the map is defined by $x=u^{2}$. The quotient is $V / K^{*}=$ Spec $K[u y]$, which is a branched cover of $X / K^{*}$, since $x y^{2}=(u y)^{2}$.

The following lemma shows that locally toric $K^{*}$-actions are ubiquitous. We note that it can be proven with fewer assumptions; see [81, [53].

Lemma 1.7.3. Let $W$ be a nonsingular variety with a relatively affine $K^{*}$-action, that is, the scheme $W / / K^{*}$ exists and the morphism $W \rightarrow W / / K^{*}$ is an affine morphism. Then the action of $K^{*}$ on $W$ is locally toric.

Proof. Taking an affine open set in $W / / K^{*}$, we may assume that $W$ is affine. We embed $W$ equivariantly into a projective space and take its completion (see, e.g., 75]). After applying equivariant resolution of singularities to this completion (see section 1.2 we may also assume that $\bar{W}$ is a nonsingular projective variety with a $K^{*}$-action, and $W \subset \bar{W}$ is an affine invariant open subset.

Let $p \in W$ be a closed point. Since $\bar{W}$ is complete, the orbit of $p$ has a limit point $q=\lim _{t \rightarrow 0} t(p)$ in $\bar{W}$. Now $q$ is fixed by $K^{*}$, hence $K^{*}$ acts on the cotangent space $m_{q} / m_{q}^{2}$ at $q$. Since $K^{*}$ is reductive, we can lift a set of eigenvectors of this 
action to semi-invariant local parameters $x_{1}, \ldots, x_{n}$ at $q$. These local parameters define a $K^{*}$-equivariant étale morphism $\eta_{q}: V_{q} \rightarrow X_{q}$ from an affine $K^{*}$ invariant open neighborhood $V_{q}$ of $q$ to the tangent space $X_{q}=\operatorname{Spec}\left(\operatorname{Sym} m_{q} / m_{q}^{2}\right)$ at $q$. The latter has a structure of a toric variety, where the torus is the complement of the zero set of $\prod x_{i}$.

Separating the parameters $x_{i}$ into $K^{*}$-invariants and noninvariants, we get a factorization $X_{q}=X_{q}^{0} \times X_{q}^{1}$, where the action of $K^{*}$ on $X_{q}^{1}$ is trivial and the action on $X_{q}^{0}$ has 0 as its unique fixed point. Thus we get a product decomposition $X_{q} / / K^{*}=X_{q}^{0} / / K^{*} \times X_{q}^{1}$.

By Luna's Fundamental Lemma ([51], Lemme 3), there exist affine $K^{*}$-invariant neighborhoods $V_{q}^{\prime}$ of $q$ and $X_{q}^{\prime}$ of 0 , such that the restriction $\eta_{q}^{\prime}: V_{q}^{\prime} \rightarrow X_{q}^{\prime}$ is strongly étale. Consider first the case $q \in W$, in which case we may replace $p$ by $q$. Denote $Z=X_{q}^{K^{*}} \cap X_{q}^{\prime}$. Then $Z \subset X_{q}^{K^{*}} \simeq X_{q}^{1}$ is affine open, and, using the direct product decomposition above, $X_{q}^{0} \times Z \subset X_{q}$ is affine open. Denote $X_{q}^{\prime \prime}=X_{q}^{\prime} \cap X_{q}^{0} \times Z$. This is affine open in $X_{q}$, and it is easy to see that $X_{q}^{\prime \prime} / / K^{*} \rightarrow X_{q} / / K^{*}$ is an open embedding: an orbit in $X_{q}^{\prime \prime}$ is closed if and only if it is closed in $X_{q}$. Writing $V_{q}^{\prime \prime}=\eta_{q}^{\prime-1} X_{q}^{\prime \prime}$, it follows that $V_{q}^{\prime \prime} \rightarrow X_{q}$ is a strongly étale toric chart.

In the case $q \notin W$, replace $V_{q}$ by $V_{q}^{\prime \prime}$. Now $\eta_{q}$ is injective on any orbit, and therefore it is injective on the orbit of $p$. Let $X_{p} \subset X_{q}$ be the affine open toric subvariety in which the torus orbit of $\eta_{q}(p)$ is closed, and let $V_{p}=\eta_{q}^{-1} X_{p} \cap W$. Now consider the restriction $\eta: V_{p} \rightarrow X_{p}$, where the $K^{*}$-orbits of $p$ and $\eta(p)$ are closed. By Luna's Fundamental Lemma there exist affine open $K^{*}$-invariant neighborhoods $V_{p}^{\prime} \subset V_{p}$ and $X_{p}^{\prime} \subset X_{p}$ of $\eta_{p}(p)$ such that the restriction $\eta: V_{p}^{\prime} \rightarrow X_{p}^{\prime}$ is a strongly étale morphism. Since $X_{p} / K^{*}$ is a geometric quotient, we have an open embedding $X_{p}^{\prime} / K^{*} \subset X_{p} / K^{*}$ and we have a strongly étale toric chart $V_{p} \rightarrow X_{p}$.

It remains to show that the charts can be chosen saturated with respect to the projection $\pi: W \rightarrow W / / K^{*}$. If the orbit of $p$ has a limit point $q=\lim _{t \rightarrow 0} t \cdot p$ or $q=\lim _{t \rightarrow \infty} t \cdot p$ in $W$, which is necessarily unique as $\pi$ is affine, then an equivariant toric chart at $q$ also covers $p$. So we may replace $p$ by $q$ and assume that the orbit of $p$ is closed. Now $\pi\left(W-V_{p}\right)$ is closed and does not contain $\pi(p)$, so we can choose an affine neighborhood $Y$ in its complement, and replace $V_{p}$ by $\pi^{-1} Y$.

\section{Birational COBORDisms}

\subsection{Definitions.}

Definition 2.1.1 (81]). Let $\phi: X_{1} \rightarrow X_{2}$ be a birational map between two algebraic varieties $X_{1}$ and $X_{2}$ over $K$, isomorphic on an open set $U$. A normal algebraic variety $B$ is called a birational cobordism for $\phi$ and denoted by $B_{\phi}\left(X_{1}, X_{2}\right)$ if it satisfies the following conditions:

(1) The multiplicative group $K^{*}$ acts effectively on $B=B_{\phi}\left(X_{1}, X_{2}\right)$.

(2) The sets

$$
\begin{aligned}
B_{-} & :=\left\{x \in B: \lim _{t \rightarrow 0} t(x) \text { does not exist in } B\right\} \\
\text { and } B_{+} & :=\left\{x \in B: \lim _{t \rightarrow \infty} t(x) \text { does not exist in } B\right\}
\end{aligned}
$$

are nonempty Zariski open subsets of $B$.

(3) There are isomorphisms

$$
B_{-} / K^{*} \stackrel{\sim}{\rightarrow} X_{1} \quad \text { and } \quad B_{+} / K^{*} \stackrel{\sim}{\rightarrow} X_{2}
$$


(4) Considering the rational map $\psi: B_{-} \rightarrow B_{+}$induced by the inclusions $\left(B_{-} \cap B_{+}\right) \subset B_{-}$and $\left(B_{-} \cap B_{+}\right) \subset B_{+}$, the following diagram commutes:

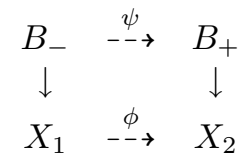

We say that $B$ respects the open set $U$ if $U$ is contained in the image of $\left(B_{-} \cap B_{+}\right) / K^{*}$.

Definition 2.1.2 (81]). Let $B=B_{\phi}\left(X_{1}, X_{2}\right)$ be a birational cobordism, and let $F \subset B^{K^{*}}$ be a subset of the fixed-point set. We define

$$
\begin{aligned}
& F^{+}=\left\{x \in B \mid \lim _{t \rightarrow 0} t(x) \in F\right\}, \\
& F^{-}=\left\{x \in B \mid \lim _{t \rightarrow \infty} t(x) \in F\right\}, \\
& F^{ \pm}=F^{+} \cup F^{-} .
\end{aligned}
$$

Definition 2.1.3 (81]). Let $B=B_{\phi}\left(X_{1}, X_{2}\right)$ be a birational cobordism. We define a relation $\prec$ among connected components of $B^{K^{*}}$ as follows: let $F_{1}, F_{2} \subset B^{K^{*}}$ be two connected components, and set $F_{1} \prec F_{2}$ if there is a point $x \notin B^{K^{*}}$ such that $\lim _{t \rightarrow 0} t(x) \in F_{1}$ and $\lim _{t \rightarrow \infty} t(x) \in F_{2}$.

Definition 2.1.4. A birational cobordism $B=B_{\phi}\left(X_{1}, X_{2}\right)$ is said to be quasielementary if any two connected components $F_{1}, F_{2} \subset B^{K^{*}}$ are incomparable with respect to $\prec$.

Note that this condition prohibits, in particular, the existence of a "loop", namely a connected component $F$ and a point $y \notin F$ such that both $\lim _{t \rightarrow 0} t(x) \in F$ and $\lim _{t \rightarrow \infty} t(x) \in F$.

Definition 2.1.5 (1] $)$. A quasi-elementary cobordism $B$ is said to be elementary if the fixed point set $B^{K^{*}}$ is connected.

Definition 2.1.6 (cf. [56], 81]). We say that a birational cobordism

$$
B=B_{\phi}\left(X_{1}, X_{2}\right)
$$

is collapsible if the relation $\prec$ is a strict pre-order, namely, there is no cyclic chain of fixed point components

$$
F_{1} \prec F_{2} \prec \ldots \prec F_{m} \prec F_{1} .
$$

2.2. The main example. We now recall a fundamental example of an elementary birational cobordism in the toric setting, discussed in [81]:

Example 2.2.1. Let $B=\mathbb{A}^{n}=\operatorname{Spec} K\left[z_{1}, \ldots, z_{n}\right]$ and let $t \in K^{*}$ act by

$$
t\left(z_{1}, \ldots, z_{i}, \ldots, z_{n}\right)=\left(t^{\alpha_{1}} z_{1}, \ldots, t^{\alpha_{i}} z_{i}, \ldots, t^{\alpha_{n}} z_{n}\right) .
$$

We assume $K^{*}$ acts effectively, namely $\operatorname{gcd}\left(\alpha_{1}, \ldots, \alpha_{n}\right)=1$. We regard $\mathbb{A}^{n}$ as a toric variety defined by a lattice $N \cong \mathbb{Z}^{n}$ and a nonsingular cone $\sigma \in N_{\mathbb{R}}$ generated by the standard basis

$$
\sigma=\left\langle v_{1}, \ldots, v_{n}\right\rangle .
$$

The dual cone $\sigma^{\vee}$ is generated by the dual basis $v_{1}^{*}, \ldots, v_{n}^{*}$, and we identify $z^{v_{i}^{*}}=z_{i}$. The $K^{*}$-action then corresponds to a one-parameter subgroup

$$
a=\left(\alpha_{1}, \ldots, \alpha_{n}\right) \in N \text {. }
$$


We assume that $a \notin \pm \sigma$. We have the obvious description of the sets $B_{+}$and $B_{-}$:

$$
\begin{aligned}
& B_{-}=\left\{\left(z_{1}, \ldots, z_{n}\right) ; z_{i} \neq 0 \text { for some } i \text { with } \alpha_{i}=\left(v_{i}^{*}, a\right)<0\right\}, \\
& B_{+}=\left\{\left(z_{1}, \ldots, z_{n}\right) ; z_{i} \neq 0 \text { for some } i \text { with } \alpha_{i}=\left(v_{i}^{*}, a\right)>0\right\} .
\end{aligned}
$$

We define the upper boundary and lower boundary fans of $\sigma$ to be

$$
\begin{aligned}
& \partial_{-} \sigma=\{x \in \sigma ; x+\epsilon \cdot a \notin \sigma \text { for all } \epsilon>0\}, \\
& \partial_{+} \sigma=\{x \in \sigma ; x+\epsilon \cdot(-a) \notin \sigma \text { for all } \epsilon>0\} .
\end{aligned}
$$

Then we obtain the description of $B_{+}$and $B_{-}$as the toric varieties corresponding to the fans $\partial_{+} \sigma$ and $\partial_{-} \sigma$ in $N_{\mathbb{R}}$.

Let $\pi: N_{\mathbb{R}} \rightarrow N_{\mathbb{R}} / \mathbb{R} \cdot a$ be the projection. Then $B / / K^{*}$ is again an affine toric variety defined by the lattice $\pi(N)$ and cone $\pi(\sigma)$. Similarly, one can check that the geometric quotients $B_{-} / K^{*}$ and $B_{+} / K^{*}$ are toric varieties defined by fans $\pi\left(\partial_{+} \sigma\right)$ and $\pi\left(\partial_{-} \sigma\right)$. Since both $\pi\left(\partial_{+} \sigma\right)$ and $\pi\left(\partial_{-} \sigma\right)$ are subdivisions of $\pi(\sigma)$, we get a diagram of birational toric maps

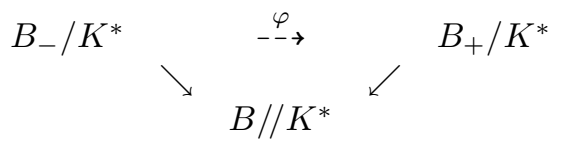

It is easy to see (see, e.g., 81]) that the varieties $B_{ \pm} / K^{*}$ have only abelian quotient singularities. Moreover, the map $\phi$ can be factored as a weighted blowing up followed by a weighted blowing down.

More generally, one can prove that if $\Sigma$ is a subdivision of a convex polyhedral cone in $N_{\mathbb{R}}$ with lower boundary $\partial_{-} \Sigma$ and upper boundary $\partial_{+} \Sigma$ relative to an element $a \in N \backslash \pm \Sigma$, then the toric variety corresponding to $\Sigma$, with the $K^{*}$-action given by the one-parameter subgroup $a \in N$, is a birational cobordism between the two toric varieties corresponding to $\pi\left(\partial_{-} \Sigma\right)$ and $\pi\left(\partial_{+} \Sigma\right)$ as fans in $N_{\mathbb{R}} / \mathbb{R} \cdot a$.

For the details, we refer the reader to [56], 81] and [4].

2.3. Construction of a cobordism. It was shown in [81] that birational cobordisms exist for a large class of birational maps $X_{1} \rightarrow X_{2}$. Here we deal with a very special case.

Theorem 2.3.1. Let $\phi: X_{1} \rightarrow X_{2}$ be a projective birational morphism between complete nonsingular algebraic varieties, which is an isomorphism on an open set $U$. Then there is a complete nonsingular algebraic variety $\bar{B}$ with an effective $K^{*}$ action, satisfying the following properties:

(1) There exist closed embeddings $\iota_{1}: X_{1} \hookrightarrow \bar{B}^{K^{*}}$ and $\iota_{2}: X_{2} \hookrightarrow \bar{B}^{K^{*}}$ with disjoint images.

(2) The open subvariety $B=\bar{B} \backslash\left(\iota_{1}\left(X_{1}\right) \cup \iota_{2}\left(X_{2}\right)\right)$ is a birational cobordism between $X_{1}$ and $X_{2}$ respecting the open set $U$.

(3) There is a coherent sheaf $E$ on $X_{2}$, with a $K^{*}$-action, and a closed $K^{*}$ equivariant embedding $\bar{B} \subset \mathbb{P}(E):=\operatorname{Proj}_{X_{2}} \operatorname{Sym} E$.

Proof. Let $J \subset \mathcal{O}_{X_{2}}$ be an ideal sheaf such that $\phi: X_{1} \rightarrow X_{2}$ is the blowing up morphism of $X_{2}$ along $J$ and $J_{U}=\mathcal{O}_{U}$. Let $I_{0}$ be the ideal of the point $0 \in \mathbb{P}^{1}$. Consider $W_{0}=X_{2} \times \mathbb{P}^{1}$ and let $p: W_{0} \rightarrow X_{2}$ and $q: W_{0} \rightarrow \mathbb{P}^{1}$ be the projections. Let $I=\left(p^{-1} J+q^{-1} I_{0}\right) \mathcal{O}_{W_{0}}$. Let $W$ be the blowing up of $W_{0}$ along $I$. (Paolo Aluffi has pointed out that this $W$ is used when constructing the deformation to the normal cone of $J$.) 
We claim that $X_{1}$ and $X_{2}$ lie in the nonsingular locus of $W$. For $X_{2} \cong X_{2} \times$ $\{\infty\} \subset X_{2} \times \mathbb{A}^{1} \subset W$ this is clear. Since $X_{1}$ is nonsingular, embedded in $W$ as the strict transform of $X_{2} \times\{0\} \subset X_{2} \times \mathbb{P}^{1}$, to prove that $X_{1}$ lies in the nonsingular locus it suffices to prove that $X_{1}$ is a Cartier divisor in $W$. We look at local coordinates. Let $A=\Gamma\left(V, \mathcal{O}_{V}\right)$ for some affine open subset $V \subset X_{2}$, and let $y_{1}, \ldots, y_{m}$ be a set of generators of $J$ on $V$. Then on the affine open subset $V \times \mathbb{A}^{1} \subset X_{2} \times \mathbb{P}^{1}$ with coordinate ring $A[x]$, the ideal $I$ is generated by $y_{1}, \ldots, y_{m}, x$. The charts of the blowing up containing the strict transform of $\{x=0\}$ are of the form

$$
\operatorname{Spec} A\left[\frac{y_{1}}{y_{i}}, \ldots, \frac{y_{m}}{y_{i}}, \frac{x}{y_{i}}\right]=\operatorname{Spec} A\left[\frac{y_{1}}{y_{i}}, \ldots, \frac{y_{m}}{y_{i}}\right] \times \operatorname{Spec} K\left[\frac{x}{y_{i}}\right],
$$

where $K^{*}$ acts on the second factor. The strict transform of $\{x=0\}$ is defined by $\frac{x}{y_{i}}$, hence it is Cartier.

Let $\bar{B} \rightarrow W$ be a canonical resolution of singularities. Then conditions (1) and (2) are clearly satisfied. For condition (3), note that $\bar{B} \rightarrow X_{2} \times \mathbb{P}^{1}$, being a composition of blowings up of invariant ideals, admits an equivariant ample line bundle. Twisting by the pullback of $\mathcal{O}_{\mathbb{P}^{1}}(n)$ we obtain an equivariant line bundle which is ample for $\bar{B} \rightarrow X_{2}$. Replacing this by a sufficiently high power and pushing forward we get $E$.

We refer the reader to 81 for more details.

We call a variety $\bar{B}$ as in the theorem a compactified, relatively projective cobordism.

2.4. Collapsibility and projectivity. Let $B=B_{\phi}\left(X_{1}, X_{2}\right)$ be a birational cobordism. We seek a criterion for collapsibility of $B$.

Let $\mathcal{C}$ be the set of connected components of $B_{\phi}\left(X_{1}, X_{2}\right)^{K^{*}}$, and let $\chi: \mathcal{C} \rightarrow \mathbb{Z}$ be a function. We say that $\chi$ is strictly increasing if $F \prec F^{\prime} \Rightarrow \chi(F)<\chi\left(F^{\prime}\right)$. The following lemma is obvious:

Lemma 2.4.1. Assume there exists a strictly increasing function $\chi$. Then $\prec$ is a strict pre-order, and $B$ is collapsible. Conversely, suppose $B$ is collapsible. Then there exists a strictly increasing function $\chi$.

Remark. It is evident that every strictly increasing function can be replaced by one which induces a strict total order. However, it will be convenient for us to consider arbitrary strictly increasing functions.

Let $\chi$ be a strictly increasing function, and let $a_{1}<a_{2}<\cdots<a_{m} \in \mathbb{Z}$ be the values of $\chi$.

Definition 2.4.2. We denote

(1) $F_{a_{i}}=\bigcup\left\{F \mid \chi(F)=a_{i}\right\}$.

(2) $F_{a_{i}}^{+}=\bigcup\left\{F^{+} \mid \chi(F)=a_{i}\right\}$.

(3) $F_{a_{i}}^{-}=\bigcup\left\{F^{-} \mid \chi(F)=a_{i}\right\}$.

(4) $F_{a_{i}}^{ \pm}=\bigcup\left\{F^{ \pm} \mid \chi(F)=a_{i}\right\}$.

(5) $B_{a_{i}}=B \backslash\left(\bigcup\left\{F^{-} \mid \chi(F)<a_{i}\right\} \cup \bigcup\left\{F^{+} \mid \chi(F)>a_{i}\right\}\right)$.

The following is an immediate extension of Proposition 1 of [81].

Proposition 2.4.3. (1) $B_{a_{i}}$ is a quasi-elementary cobordism.

(2) For $i=1, \ldots, m-1$ we have $\left(B_{a_{i}}\right)_{+}=\left(B_{a_{i+1}}\right)_{-}$. 
The following is an analogue of Lemma 1 of 81 in the case of the cobordisms we have constructed.

Proposition 2.4.4. Let $E$ be a coherent sheaf on $X_{2}$ with a $K^{*}$-action, and let $\bar{B} \subset \mathbb{P}(E)$ be a compactified, relatively projective cobordism embedded $K^{*}$-equivariantly. Then there exists a strictly increasing function $\chi$ for the cobordism $B=$ $\bar{B} \backslash\left(X_{1} \cup X_{2}\right)$. In particular, the cobordism is collapsible.

Proof. Since $K^{*}$ acts trivially on $X_{2}$, and since $K^{*}$ is reductive, there exists a direct sum decomposition

$$
E=\bigoplus_{b \in \mathbb{Z}} E_{b}
$$

where $E_{b}$ is the subsheaf on which the action of $K^{*}$ is given by the character $t \mapsto t^{b}$. Denote by $b_{0}, \ldots, b_{k}$ the characters which figure in this representation. Note that there are disjoint embeddings $\mathbb{P}\left(E_{b_{j}}\right) \subset \mathbb{P}(E)$.

Let $p \in B$ be a fixed point lying in the fiber $\mathbb{P}\left(E_{q}\right)$ over $q \in X_{2}$. We choose a basis

$$
\left(x_{b_{0}, 1}, \ldots, x_{b_{0}, d_{0}}, \ldots, x_{b_{k}, 1}, \ldots, x_{b_{k}, d_{k}}\right)
$$

of $E_{q}$ where $x_{b_{j}, \nu} \in E_{b_{j}}$ and use the following lemma:

Lemma 2.4.5. Suppose $p \in \mathbb{P}\left(E_{q}\right)^{K^{*}}$ is a fixed point with homogeneous coordinates

$$
\left(p_{b_{0}, 1}, \ldots, p_{b_{0}, d_{0}}, \ldots, p_{b_{k}, 1}, \ldots, p_{b_{k}, d_{k}}\right) .
$$

Then there is a $j_{p}$ such that $p_{b_{j}, \nu}=0$ whenever $j \neq j_{p}$. In particular, $p \in \mathbb{P}\left(E_{b_{j_{p}}}\right) \subset$ $\mathbb{P}(E)$.

If $F \subset B^{K^{*}}$ is a connected component of the fixed point set, then it follows from the lemma that $F \subset \mathbb{P}\left(E_{b_{j}}\right)$ for some $j$. We define

$$
\chi(F)=b_{j} .
$$

To check that $\chi$ is strictly increasing, consider a point $p \in B$ such that $\lim _{t \rightarrow 0} t(p) \in$ $F_{1}$ and $\lim _{t \rightarrow \infty} t(p) \in F_{2}$ for some fixed point components $F_{1}$ and $F_{2}$. Let the coordinates of $p$ in the fiber over $q \in X_{2}$ be $\left(p_{b_{0}, 1}, \ldots, p_{b_{0}, d_{0}}, \ldots, p_{b_{k}, 1}, \ldots, p_{b_{k}, d_{k}}\right)$. Now

$$
\begin{aligned}
\lim _{t \rightarrow 0} t(p) & \in \mathbb{P}\left(E_{b_{\text {min }}}\right), \\
\lim _{t \rightarrow \infty} t(p) & \in \mathbb{P}\left(E_{b_{\max }}\right),
\end{aligned}
$$

where

$$
\begin{aligned}
b_{\text {min }} & =\min \left\{b_{j}: p_{b_{j}, \nu} \neq 0 \text { for some } \nu\right\}, \\
b_{\max } & =\max \left\{b_{j}: p_{b_{j}, \nu} \neq 0 \text { for some } \nu\right\} .
\end{aligned}
$$

Thus, if $p$ is not fixed by $K^{*}$, then

$$
\chi\left(F_{1}\right)=b_{\min }<b_{\max }=\chi\left(F_{2}\right) .
$$


2.5. Geometric invariant theory and projectivity. In this section we use geometric invariant theory and ideas (originating in symplectic geometry) developed by M. Thaddeus and others (see, e.g., [78]), in order to obtain a result about relative projectivity of quotients.

We continue with the notation of the last section. Consider the sheaf $E$ and its decomposition according to the character. Let $\left\{b_{j}\right\}$ be the characters of the action of $K^{*}$ on $E$ and $\left\{a_{i}\right\}$ the subset of those $b_{j}$ that are in the image of $\chi$. If we use the Veronese embedding $\bar{B} \subset \mathbb{P}\left(\operatorname{Sym}^{2}(E)\right)$ and replace $E$ by $\operatorname{Sym}^{2}(E)$, we may assume that $a_{i}$ are even, in particular $a_{i+1}>a_{i}+1$ (this is a technical condition which comes in handy in what follows).

Denote by $\rho_{0}(t)$ the action of $t \in K^{*}$ on $E$. For any $r \in \mathbb{Z}$ consider the "twisted" action $\rho_{r}(t)=t^{-r} \cdot \rho_{0}(t)$. Note that the induced action on $\mathbb{P}(E)$ does not depend on the "twist" $r$. Considering the decomposition $E=\bigoplus E_{b_{j}}$, we see that $\rho_{r}(t)$ acts on $E_{b_{j}}$ by multiplication by $t^{b_{j}-r}$.

We can apply geometric invariant theory in its relative form (see, e.g., 63], [33]) to the action $\rho_{r}(t)$ of $K^{*}$. Recall that a point $p \in \mathbb{P}(E)$ is said to be semistable with respect to $\rho_{r}$, written $p \in\left(\mathbb{P}(E), \rho_{r}\right)^{s s}$, if there is a positive integer $n$ and a $\rho_{r}$-invariant local section $s \in\left(\operatorname{Sym}^{n}(E)\right)^{\rho_{r}}$, such that $s(p) \neq 0$. The main result of geometric invariant theory implies that

$$
\underset{X_{2}}{\mathcal{P} r o j} \bigoplus_{n \geq 0}^{\infty}\left(\operatorname{Sym}^{n}(E)\right)^{\rho_{r}}=\left(\mathbb{P}(E), \rho_{r}\right)^{s s} / / K^{*}
$$

moreover, the quotient map $\left(\mathbb{P}(E), \rho_{r}\right)^{s s} \rightarrow\left(\mathbb{P}(E), \rho_{r}\right)^{s s} / / K^{*}$ is affine. We can define $\left(\bar{B}, \rho_{r}\right)^{s s}$ analogously, and we automatically have $\left(\bar{B}, \rho_{r}\right)^{s s}=\bar{B} \cap\left(\mathbb{P}(E), \rho_{r}\right)^{s s}$.

The numerical criterion of semistability (see [60]) immediately implies the following:

Lemma 2.5.1. For $0 \leq i \leq m$ we have

(1) $\left(\bar{B}, \rho_{a_{i}}\right)^{s s}=B_{a_{i}}$.

(2) $\left(\bar{B}, \rho_{a_{i}+1}\right)^{s s}=\left(B_{a_{i}}\right)_{+}$.

(3) $\left(\bar{B}, \rho_{a_{i}-1}\right)^{s s}=\left(B_{a_{i}}\right)_{-}$.

In other words, the triangle of birational maps

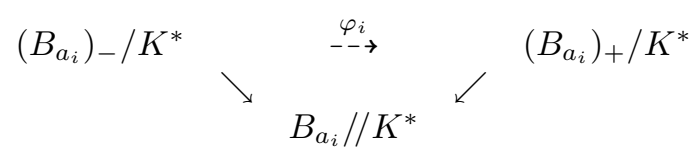

is induced by a change of linearization of the action of $K^{*}$.

In particular we obtain:

Proposition 2.5.2. The morphisms $\left(B_{a_{i}}\right)_{+} / K^{*} \rightarrow X_{2},\left(B_{a_{i}}\right)_{-} / K^{*} \rightarrow X_{2}$ and $B_{a_{i}} / / K^{*} \rightarrow X_{2}$ are projective.

2.6. The main result of [81]. Let $B$ be a collapsible nonsingular birational cobordism. Then we can write $B$ as a union of quasi-elementary cobordisms $B=\bigcup_{i} B_{a_{i}}$, with $\left(B_{a_{i}}\right)_{+}=\left(B_{a_{i+1}}\right)_{-}$. By Lemma 1.7 .3 each $B_{a_{i}}$ has a locally toric structure such that the action of $K^{*}$ is locally toric.

Lemma 2.6.1. Let $B_{a_{i}}$ be a quasi-elementary cobordism, with a relatively affine locally toric $K^{*}$-action. Then $B_{a_{i}} / / K^{*},\left(B_{a_{i}}\right)_{-} / K^{*},\left(B_{a_{i}}\right)_{+} / K^{*}$ are locally toric 
varieties and we have a diagram of locally toric maps

$$
\left(B_{a_{i}}\right)_{-} / K^{*} \underset{\substack{\varphi_{i} \\ \stackrel{--\rightarrow}{ }}}{B_{a_{i}} / / K^{*}} \swarrow^{\left(B_{a_{i}}\right)_{+} / K^{*}}
$$

where $\varphi_{i}$ is a tightly locally toric birational transformation.

In case $B_{a_{i}}$ is nonsingular, the diagram above can be described in toric charts by the main example in section 2.2.

If the action of $K^{*}$ on $B_{a_{i}}$ is toroidal, then all these varieties and maps are also toroidal, and $\varphi_{i}$ is a toroidal birational map.

Proof. Let $\eta_{p}: V_{p} \rightarrow X_{p}$ be a strongly étale $K^{*}$-equivariant toric chart in $B_{a_{i}}$ giving a locally toric structure to the action of $K^{*}$. Then $\left(V_{p}\right)_{-}=\left(B_{a_{i}}\right)_{-} \cap V_{p}$ and the morphism $\left(V_{p}\right)_{-} \rightarrow\left(X_{p}\right)_{-}$is again strongly étale, providing locally toric structures on the variety $\left(B_{a_{i}}\right)_{-} / K^{*}$ and the morphism $\left(B_{a_{i}}\right)_{-} / K^{*} \rightarrow B_{a_{i}} / / K^{*}$. Similarly for $\left(B_{a_{i}}\right)_{+}$.

Now we assume $B \subset \bar{B}$ is open in a compactified, relatively projective cobordism. When we compose the birational transformations obtained from each $B_{a_{i}}$ we get a slight refinement of the main result of [81].

Theorem 2.6.2. Let $\phi: X_{1} \rightarrow X_{2}$ be a birational map between complete nonsingular algebraic varieties $X_{1}$ and $X_{2}$ over an algebraically closed field $K$ of characteristic zero, and let $U \subset X_{1}$ be an open set where $\phi$ is an isomorphism. Then there exists a sequence of birational maps between complete locally toric algebraic varieties

$$
X_{1}=W_{0} \stackrel{\varphi_{1}}{\rightarrow} W_{1} \stackrel{\varphi_{2}}{\rightarrow} \cdots \stackrel{\varphi_{i-1}}{\rightarrow} W_{i-1} \stackrel{\varphi_{i}}{\rightarrow} W_{i} \stackrel{\varphi_{i+1}}{\rightarrow} \ldots \stackrel{\varphi_{m-1}}{\rightarrow-\rightarrow} W_{m-1} \stackrel{\varphi_{m}}{\rightarrow} W_{m}=X_{2}
$$

where

(1) $\phi=\varphi_{m} \circ \varphi_{m-1} \circ \cdots \circ \varphi_{2} \circ \varphi_{1}$,

(2) $\varphi_{i}$ are isomorphisms on $U$, and

(3) for each $i$, the birational transformation $\varphi_{i}$ is tightly locally toric and étale locally equivalent to a map $\varphi$ described in 2.2. In particular $W_{i}$ have finite abelian quotient singularities, and $\varphi_{i}$ can be obtained as a weighted blowing up followed by a weighted blowing down.

Furthermore, there is an index $i_{0}$ such that for all $i \leq i_{0}$ the map $W_{i} \rightarrow X_{1}$ is a projective morphism, and for all $i \geq i_{0}$ the map $W_{i} \rightarrow X_{2}$ is a projective morphism. In particular, if $X_{1}$ and $X_{2}$ are projective, then all the $W_{i}$ are projective.

Remark. For the projectivity claim (2), we take the first $i_{0}$ terms in the factorization to come from Hironaka's elimination of indeterminacies in Lemma 1.3.1, which is projective over $X_{1}$, whereas the last terms come from $\bar{B}$, which is projective over $X_{2}$, and the geometric invariant theory considerations as in Proposition 2.5.2.

2.7. Projectivity of toroidal weak factorization. The following is a refinement of Theorem 1.6.1 in which a projectivity statement is added:

Theorem 2.7.1. Let $U_{1} \subset W_{1}$ and $U_{2} \subset W_{2}$ be nonsingular toroidal embeddings. Let $\psi: W_{1} \rightarrow W_{2}$ be a proper toroidal birational map. Then $\phi$ can be factored into a sequence of toroidal birational maps consisting of toroidal blowings up and down of nonsingular centers, namely:

$$
W_{1}=V_{0} \stackrel{\varphi_{1}}{-\rightarrow} V_{1} \stackrel{\varphi_{2}}{\rightarrow-} \cdots \stackrel{\varphi_{i-1}}{\rightarrow--\rightarrow} V_{i-1} \stackrel{\varphi_{i}}{\rightarrow--} V_{i} \stackrel{\varphi_{i+1}}{\rightarrow} \cdots \stackrel{\varphi_{l-1}}{\rightarrow} V_{l-1} \stackrel{\varphi_{l}}{\rightarrow} V_{l}=W_{2}
$$


where

(1) $\phi=\varphi_{l} \circ \varphi_{l-1} \circ \cdots \circ \varphi_{2} \circ \varphi_{1}$,

(2) $\varphi_{i}$ are isomorphisms on $U$, the embeddings $U \subset V_{i}$ are toroidal, and $\varphi_{i}$ are toroidal birational maps, and

(3) either $\varphi_{i}: V_{i-1} \rightarrow V_{i}$ or $\varphi_{i}^{-1}: V_{i} \rightarrow V_{i-1}$ is a toroidal morphism obtained by blowing up a nonsingular irreducible toroidal center.

Furthermore, there is an index $i_{0}$ such that for all $i \leq i_{0}$ the map $V_{i} \rightarrow W_{1}$ is a projective morphism, and for all $i \geq i_{0}$ the map $V_{i} \rightarrow W_{2}$ is a projective morphism. In particular, if $W_{1}$ and $W_{2}$ are projective, then all the $V_{i}$ are projective.

Proof. As in 4], Lemma 8.7, we reduce to the case where the polyhedral complex of $W_{2}$ is embeddable as a quasi-projective toric fan $\Delta_{2}$ in a space $N_{\mathbb{R}}$. Indeed that lemma gives an embedding preserving the $\mathbb{Q}$-structure for the barycentric subdivision of any simplicial complex, and since $\Delta_{2}$ is nonsingular this embedding preserves integral structures as well. A further subdivision ensures that the fan is quasi-projective. (We note that this embedding is introduced for the sole purpose of applying Morelli's $\pi$-desingularization lemma directly, rather than observing that the proof works word for word in the toroidal case.)

As in 1.3.1 we may assume $W_{1} \rightarrow W_{2}$ is a projective morphism. Thus the complex $\Delta_{1}$ of $W_{1}$ is a projective subdivision of $\Delta_{2}$. Our construction of a compactified relatively projective cobordism $\bar{B}$ for the morphism $\phi$ yields a toroidal embedding $B$ whose complex $\Delta_{B}$ is a quasi-projective polyhedral cobordism lying in $(N \oplus \mathbb{Z})_{\mathbb{R}}$ such that $\pi\left(\partial_{+} \Delta_{B}\right)=\Delta_{2}$ and $\pi\left(\partial_{-} \Delta_{B}\right)=\Delta_{1}$, where $\pi$ is the projection onto $N_{\mathbb{R}}$. Moreover, the toroidal morphism $B \rightarrow W_{2}$ gives a polyhedral morphism $\Delta_{B} \rightarrow \Delta_{2}$ induced by the projection $\pi$. Morelli's $\pi$-desingularization lemma gives a projective subdivision $\Delta_{B}^{\prime} \rightarrow \Delta_{B}$, isomorphic on the upper and lower boundaries $\partial_{ \pm} \Delta_{B}$, such that $\Delta_{B}^{\prime}$ is $\pi$-nonsingular. We still have a polyhedral morphism $\Delta_{B}^{\prime} \rightarrow \Delta_{2}$. The complex $\Delta_{B}^{\prime}$ corresponds to a toroidal birational cobordism $B^{\prime}$ between $W_{1}$ and $W_{2}$. Since $\Delta_{B}^{\prime}$ is $\pi$-nonsingular, any elementary piece $B_{F}^{\prime} \subset B^{\prime}$ corresponds to a toroidal blowing up followed by a toroidal blowing down between nonsingular toroidal embeddings, with nonsingular centers. It follows that the same holds for every quasi-elementary piece of $B^{\prime}$ (here the centers may be reducible, but blowing up a reducible center is the composition of blowings up of its connected components one at a time). As in Theorem 2.6.2 above, these toroidal embeddings can be chosen to be projective over $W_{2}$.

\section{TORIFICATION}

We wish to replace the locally toric factorization of Theorem 2.6 .2 by a toroidal factorization. This amounts to replacing $B$ with a locally toric $K^{*}$-action by some $B^{\prime}$ with a toroidal $K^{*}$-action. We call such a procedure torification. The basic idea, which goes back at least to Hironaka, is that if one blows up an ideal, the exceptional divisors provide the resulting variety with useful extra structure. The ideal we construct, called a torific ideal, is closely related to the torific ideal of [2].

3.1. Construction of a torific ideal. Let $B$ be a normal variety with a relatively affine $K^{*}$-action. We denote by $\pi: B \rightarrow B / / K^{*}$ the quotient morphism, which by assumption is affine.

Consider the quasi-coherent sheaf of algebras $A=\pi_{*} \mathcal{O}_{B}$ together with a $K^{*}$ action on it. For an integer $\alpha$ we denote by $A_{\alpha} \subset A$ the subsheaf of semi-invariant 
sections $f \in A$ of $K^{*}$-character $\alpha$ :

$$
t^{*}(f)=t^{\alpha} f
$$

Definition 3.1.1. The $K^{*}$-equivariant ideal sheaf $I_{\alpha}^{B}$ on $B$, generated by $A_{\alpha}$, is called the $\alpha$-torific ideal sheaf of the action of $K^{*}$.

We sometimes omit the superscript $B$ and write $I_{\alpha}$ if there is no risk of confusion.

Let $B$ be a locally toric, quasi-elementary cobordism with a relatively affine, locally toric $K^{*}$-action; $B=B_{a_{i}}$ for some $i$ according to our previous notation. We continue to denote by $\pi: B \rightarrow B / / K^{*}$ the quotient morphism.

Recall that by Definition 1.7 .2 of a locally toric action, the birational cobordism $B$ is covered with locally toric charts of the form

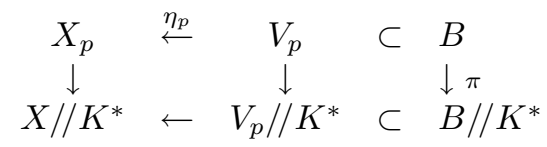

with both squares Cartesian and the horizontal maps étale. For a chart as above, let $I_{\alpha}^{X_{p}}$ be the $\alpha$-torific ideal sheaf on $X_{p}$.

Lemma 3.1.2. We have

$$
\left.I_{\alpha}^{B}\right|_{V_{p}}=\eta_{p}^{-1} I_{\alpha}^{X_{p}}
$$

Moreover, the ideal sheaf $I_{\alpha}^{X_{p}}$ is generated by monomials of $K^{*}$-character $\alpha$.

Proof. Assume that $f \in A_{\alpha}$ is regular at $p \in B$. Replacing $V_{p}$ by a smaller open set if necessary, we may assume that $f$ is regular on $V_{p}$. We have

$$
f \in \mathcal{O}\left(V_{p}\right)=\mathcal{O}\left(X_{p}\right) \otimes_{O\left(X_{p} / / K^{*}\right)} \mathcal{O}\left(V_{p} / / K^{*}\right)
$$

Now $K^{*}$ acts trivially on sections of $\mathcal{O}_{V_{p} / / K^{*}}$, hence $f$ lies in the ideal generated by pullbacks of sections of $\mathcal{O}_{X_{p}}$ of $K^{*}$-character $\alpha$. The second statement is clear.

Note that the zero function lies in every $A_{c}$, and it is conceivable that some $I_{c}$ is the zero ideal. This does not happen for a cobordism:

Lemma 3.1.3. For any $c \in \mathbb{Z}$, the ideal $I_{c}^{B}$ is nonzero.

Proof. By Lemma 3.1.2 it suffices to prove this for the ideals $I_{c}^{X_{p}}$ on the toric charts $X_{p}$. Let $X=X(N, \sigma)$ be an affine toric variety, with an effective $K^{*}$-action on $X$ given by a primitive lattice point $a \in N$. Since $X_{-} \cap X_{+}$is a nonempty open subset, it follows that $a \notin \pm \sigma$. This implies that the set of points $m \in M \cap \sigma^{\vee}$ such that $(m, a)=c$ is nonempty. Thus the set of nonzero $f \in \mathcal{O}_{X_{p}}$ of $K^{*}$-character $\alpha$ is nonempty. Hence the ideal $I_{c}^{X}$ is nonzero.

Assume further that the locally toric quasi-elementary cobordism $B$ is nonsingular, covered with a finite number of locally toric charts as above. For each chart $\eta_{p}: V_{p} \rightarrow X_{p}$ we choose monomial coordinates $z_{1}, \ldots, z_{n}$ generating $\mathcal{O}_{X_{p}}$. Let $\mathcal{C}=\left\{c_{1}, \ldots, c_{\mu}\right\}$ be a finite set of integers containing the characters of $K^{*}$-action on the coordinates $z_{i}$ for all charts. Let

$$
I=I^{B}=I_{c_{1}}^{B} \cdots I_{c_{\mu}}^{B}
$$

be the product of the $c_{i}$-torific ideals, and let $B^{\text {tor }} \rightarrow B$ be the normalized blowing up of $B$ along $I$. Since $I$ is $K^{*}$-equivariant, the action of $K^{*}$ lifts to $B^{t o r}$. Denote by $D \subset B^{\text {tor }}$ the total transform of the zero set of $I$, and $U_{B^{t o r}}=B^{\text {tor }} \backslash D$. 
Definition 3.1.4. We call $I^{B}$ a torific ideal and $B^{\text {tor }} \rightarrow B$ a torific blowing up.

It follows that $B^{\text {tor }}$, being the normalized blowing up of the product $I_{c_{1}} \cdots I_{c_{\mu}}$, satisfies a universal property: it is the minimal normal modification of $B$ such that the inverse image of $I_{c_{i}}$ is principal for all $i$. This implies that $B^{\text {tor }}$ is canonically isomorphic to the normalization of the variety obtained from $B$ by first blowing up $I_{c_{1}}$, then the inverse image of $I_{c_{2}}$, and so on.

3.2. The torifying property of the torific ideal. To justify the terminology of Definition 3.1.4 we are going to show that $U_{B^{t o r}} \subset B^{\text {tor }}$ is a toroidal embedding on which $K^{*}$ acts toroidally. It clearly suffices to prove this for the toric varieties $X_{p}^{\text {tor }}$ obtained by blowing up the locally toric charts $X_{p}$ along monomial ideals $I^{X_{p}}$. We are thus led to the following problem: given a toric variety $X$ with a $K^{*}$-action and a divisor $D \subset X \backslash T$, when are the embedding $X \backslash D \subset X$ and the $K^{*}$-action on it toroidal?

In this situation we find it useful to keep in mind the pair $(X, D)$ instead of the open embedding $X \backslash D \subset X$. Denote by $D_{X}$ the reduced Weil divisor $X \backslash T$, and write $D_{X}=D \cup D^{\prime}$. Following [3], section 3, we say that $(X, D)$ is obtained by removing the divisor $D^{\prime}$ from the toroidal structure $\left(X, D_{X}\right)$. Therefore the question above can be rephrased as follows: which reduced Weil divisors $D^{\prime} \subset D_{X}$ can be removed from the toroidal structure so that the resulting pair is toroidal, with toroidal $K^{*}$-action?

Example 3.2.1. Consider the affine line $X=\operatorname{Spec} K[x]$, a toric variety with the standard $K^{*}$-action $x \mapsto t x, \quad t \in K^{*}$, and toric divisor $D=D_{X}=\{x=0\}$. The pair $(X, D)$ is toroidal and the action of $K^{*}$ is toroidal. The pair $(X, \emptyset)$ is also toroidal, but the action on this pair is not toroidal.

Example 3.2.2. Consider the affine plane $X=\operatorname{Spec} K[x, y]$, a toric variety with toric divisor $D_{X}=\{x y=0\}$. Consider the $K^{*}$-action $(x, y) \mapsto(t x, y), \quad t \in K^{*}$. If we denote $D=\{x=0\}, D^{\prime}=\{y=0\}$, the pair $(X, D)$ is toroidal and the action of $K^{*}$ is toroidal. Thus the divisor $D^{\prime}$ can be removed from the standard toroidal structure $\left(X, D_{X}\right)$ keeping the action toroidal.

We start with some combinatorics. Let $X=X(N, \sigma)$ be an affine toric variety. If $\rho \subset \sigma^{\vee}$ is a face of $\sigma^{\vee}$, we say that $\rho$ splits off from $\sigma^{\vee}$ with complement $\tau$ if we have

$$
\begin{aligned}
& \sigma^{\vee} \cong \tau \times \rho, \\
& M \cong M_{\tau} \times M_{\rho},
\end{aligned}
$$

where $\tau \subset \sigma^{\vee}$ is a subcone, not necessarily a face, and $M_{\tau}$ (resp. $M_{\rho}$ ) is the sublattice of $M$ generated by $M \cap \tau$ (resp. $M \cap \rho$ ).

Lemma 3.2.3. Let $\rho_{1}, \ldots, \rho_{k}$ be a subset of the codimension 1 faces of $\sigma^{\vee}$, and let $w_{1}, \ldots, w_{k} \in M \cap \sigma^{\vee}$. The following are equivalent:

(1) For each $i=1, \ldots, k$ the face $\rho_{i}$ splits off from $\sigma^{\vee}$ with complement $\left\langle w_{i}\right\rangle$ :

$$
\begin{aligned}
& \sigma^{\vee} \cong\left\langle w_{i}\right\rangle \times \rho_{i}, \\
& M \cong \mathbb{Z} w_{i} \times M_{\rho_{i}} .
\end{aligned}
$$


(2) $w_{1}, \ldots, w_{k}$ are linearly independent, generating a unimodular sublattice of $M$, and the face $\rho_{1} \cap \ldots \cap \rho_{k}$ splits off from $\sigma^{\vee}$ with complement $\left\langle w_{1}, \ldots, w_{k}\right\rangle$ :

$$
\begin{aligned}
\sigma^{\vee} & \cong\left\langle w_{1}, \ldots, w_{k}\right\rangle \times \rho_{1} \cap \ldots \cap \rho_{k}, \\
M & \cong \mathbb{Z} w_{1} \times \cdots \times \mathbb{Z} w_{k} \times M_{\rho_{1} \cap \ldots \cap \rho_{k}} .
\end{aligned}
$$

Proof. The implication $(1) \Leftarrow(2)$ is trivial. The converse follows by induction on $k$. One writes $\sigma^{\vee} \cong\left\langle w_{1}\right\rangle \times \rho_{1}$ and shows that for all $i=2, \ldots, k$ the face $\rho_{1} \cap \rho_{i}$ splits off from $\rho_{1}$ with complement $\left\langle w_{i}\right\rangle$.

The geometric content of the lemma is the following:

Lemma 3.2.4. Let $X$ be an affine toric variety with $D_{1}, \ldots, D_{k}, E_{1}, \ldots, E_{l}$ the irreducible toric divisors of $X$. Assume that $D_{1}, \ldots, D_{k}$ are Cartier and let $z^{w_{1}}, \ldots$, $z^{w_{k}}$ be a set of monomials defining these divisors. The following are equivalent:

(1) For each $i=1, \ldots, k$ we can write $X$ as a product of toric varieties

$$
\begin{aligned}
X & \cong \operatorname{Spec} K\left[z^{w_{i}}\right] \times X_{i} \cong \mathbb{A}^{1} \times X_{i}, \\
D_{i} & \cong\{0\} \times X_{i} .
\end{aligned}
$$

(2) We can write $X$ as a product of toric varieties

$$
\begin{aligned}
& X \cong \operatorname{Spec} K\left[z^{w_{1}}, \ldots, z^{w_{k}}\right] \times X^{\prime} \cong \mathbb{A}^{k} \times X^{\prime}, \\
& D_{i} \cong D_{i}^{\prime} \times X^{\prime}
\end{aligned}
$$

where $D_{i}^{\prime}$ are the irreducible toric divisors in $\mathbb{A}^{k}$ defined by $z^{w_{i}}$.

If these conditions are satisfied, then $\left(X, \bigcup_{i} E_{i}\right)$ is a toroidal pair, i.e.,

$$
\left(X \succ \bigcup_{i} E_{i}\right) \subset X
$$

is a toroidal embedding. If, moreover, $K^{*}$ acts on $X$ as a subgroup of the torus and $z^{w_{i}}$ are invariant for all $i=1, \ldots, k$, then $K^{*}$ acts toroidally on this embedding.

Proof. The equivalence of the two conditions follows from the previous lemma. (Note that because $z^{w_{i}}$ define distinct divisors $D_{i}$, the complementary faces $\rho_{i}$ of $\sigma^{\vee}$ are distinct.) For the last statements it suffices to cover $X \cong \mathbb{A}^{k} \times X^{\prime}$ with charts of the form $\mathbb{G}_{m}^{k} \times X^{\prime}$.

Note that $\left(X, \bigcup_{i} E_{i}\right)$ in the lemma is the toroidal pair obtained from $X$ by removing the divisors $D_{1}, \ldots, D_{k}$ from the toroidal structure $\left(X,\left(\bigcup_{i} E_{i}\right) \cup\left(\bigcup_{j} D_{j}\right)\right)$.

We are now ready to prove the main result of this section. Recall that $B$ is a nonsingular quasi-elementary birational cobordism, with relatively affine $K^{*}$-action, and $p: B^{\text {tor }} \rightarrow B$ is the torifying blowup constructed in section 3.1.

Proposition 3.2.5. (1) The variety $B^{\text {tor }}$ is a quasi-elementary cobordism, with $\left(B^{\text {tor }}\right)_{+}=B^{\text {tor }} \times_{B} B_{+}$and $\left(B^{\text {tor }}\right)_{-}=B^{\text {tor }} \times_{B} B_{-}$.

(2) The embedding $U_{B^{\text {tor }}} \subset B^{\text {tor }}$ is toroidal and $K^{*}$ acts toroidally on this embedding.

Proof. Let us first see that the action of $K^{*}$ on $B^{\text {tor }}$ is relatively affine, which also implies that $B^{\text {tor }}$ is quasi-elementary: otherwise the closure of a $K^{*}$-orbit is a complete rational curve, which cannot be contained in the fiber of an affine morphism. 
The ideal $I$ is defined as $I_{c_{1}} \cdots I_{c_{\mu}}$ for some finite set of characters $\left\{c_{1}, \ldots, c_{\mu}\right\}$. Each $I_{c_{i}}$ is the ideal generated by $A_{c_{i}}$, the subsheaf of $\pi_{*} \mathcal{O}_{B}$ of functions with $K^{*}$ character $c_{i}$. Therefore $I$ is generated by $J:=A_{c_{1}} \cdots A_{c_{\mu}}$, which is a coherent sheaf on $B / / K^{*}$. We thus have a surjective sheaf homomorphism $J \otimes_{\mathcal{O}_{B / / K^{*}}} \mathcal{O}_{B} \rightarrow I$, inducing a closed embedding

$$
\underset{B}{\operatorname{Proj}}\left(\bigoplus_{k \geq 0} I^{k}\right) \subset \quad B \underset{B / / K^{*}}{\times} \underset{B / / K^{*}}{\mathcal{P} r o j}\left(\bigoplus_{k \geq 0} J^{\otimes k}\right) .
$$

Since the normalization morphism is finite and the quotient morphism $B \rightarrow B / / K^{*}$ is affine, it follows that $B^{\text {tor }} \rightarrow \mathcal{P} \operatorname{roj}_{B / / K^{*}}\left(\bigoplus_{k \geq 0} J^{\otimes k}\right)$ is an affine invariant morphism, showing that the action is relatively affine.

We note that $\left(B^{\text {tor }}\right)^{K^{*}}$ is the inverse image of $B^{K^{*}}$. For this it suffices to show that the fiber of $B^{\text {tor }} \rightarrow B$ over a fixed point consists of fixed points. This follows since the coordinate ring of an affine chart in a $K^{*}$-invariant fiber of the morphism $B^{\text {tor }} \rightarrow B$ is generated by fractions $f=f_{1} / f_{2}$ where $f_{i}$ are generators of the ideal $I$, hence $K^{*}$ acts trivially on $f$.

Combining this with the fact that $B^{t o r} \rightarrow B$ is proper, we get that $x \in\left(B^{t o r}\right)_{+}$ if and only if its image is in $B_{+}$, and similarly for $\left(B^{\text {tor }}\right)_{-}$. This proves the first part of the proposition. For the same reason, if an open set $V \subset B$ is saturated (i.e., $\left.V=\pi^{-1} \pi(V)\right)$, then the same holds for its inverse image $V^{\text {tor }} \subset B^{\text {tor }}$.

To prove that $U_{B^{\text {tor }}} \subset B^{\text {tor }}$ is toroidal and $K^{*}$ acts toroidally on this embedding, we consider toric charts $\eta_{p}: V_{p} \rightarrow X_{p}$ in $B$ giving the action of $K^{*}$ on $B$ a locally toric structure. For simplicity we write $V=V_{p}, X=X_{p}$. By Lemma 3.1.2 the ideal $I=I^{B}$ restricted to $V$ is the inverse image of the ideal $I^{X}=I_{c_{1}}^{X} \cdots I_{c_{\mu}}^{X}$ in $X$. It follows that the normalization $X^{\text {tor }}$ of the blowing up of $I^{X}$ in $X$ provides a toric chart $\eta^{\text {tor }}: V^{\text {tor }} \rightarrow X^{\text {tor }}$ for $B^{\text {tor }}$ such that the action of $K^{*}$ on $B^{\text {tor }}$ is again locally toric. Let $D^{\text {tor }} \subset X^{\text {tor }}$ be the support of the divisor defined by the total transform of $I^{X}$. Then

$$
U_{B^{t o r}} \cap V^{t o r}=\eta^{t o r-1}\left(X^{t o r}-D^{t o r}\right),
$$

and we are reduced to proving that $\left(X^{t o r}>D^{t o r}\right) \subset X^{\text {tor }}$ is a toroidal embedding on which $K^{*}$ acts toroidally. In other words, we have to show that the irreducible toric divisors $D \subset X^{\text {tor }}$ that do not lie in $D^{\text {tor }}$ can be removed from the standard toroidal structure given by the toric structure, keeping the $K^{*}$-action toroidal. By Lemma 3.2.4 we can remove them one at a time.

Write $X=X(N, \sigma)$ where $\sigma=\left\langle v_{1}, \ldots, v_{m}\right\rangle, \sigma^{\vee}=\left\langle v_{1}^{*}, \ldots, v_{m}^{*}, \pm v_{m+1}^{*}, \ldots, \pm v_{n}^{*}\right\rangle$, and let $K^{*}$ act on $z_{i}=z^{v_{i}^{*}}$ by character $c_{i}$. The only irreducible toric divisors in $X^{\text {tor }}$ that do not lie in the total transform of $I^{X}$ are among the strict transforms of the divisors $\left\{z_{i}=0\right\} \subset X$. Consider the divisor $\left\{z_{1}=0\right\}$. The ideal $I_{c_{1}}^{X}$ contains $z_{1}$. If $I_{c_{1}}^{X}$ is principal, then the strict transform of $\left\{z_{1}=0\right\}$ is a component of $D^{\text {tor }}$ and there is nothing to prove. Assume that this is not the case and choose monomial generators for $I_{c_{1}}^{X}$ corresponding to lattice points $v_{1}^{*}, m_{1}, \ldots, m_{l}$ in $M \cap \sigma^{\vee}$. We may assume that $z^{m_{i}}$ are not divisible by $z_{1}$. To study the strict transform of $\left\{z_{1}=0\right\}$ in $X^{\text {tor }}$ we first blow up $I_{c_{1}}^{X}$, then the rest of the $I_{c_{i}}^{X}$, and then normalize.

Let $Y$ be an affine chart of the blowing up of $X$ along $I_{c_{1}}^{X}$ (which is not necessarily normal), obtained by inverting one of the generators of $I_{c_{1}}^{X}$, and let $D$ be the strict transform of $\left\{z_{1}=0\right\}$ in $Y$. Then $D$ is nonempty if and only if $Y$ is the chart of 
the blowing up where we invert one of the $z^{m_{i}}$, say $z^{m_{1}}$. We have

$$
\begin{aligned}
Y & =\operatorname{Spec} K\left[\frac{z_{1}}{z^{m_{1}}}, \frac{z^{m_{2}}}{z^{m_{1}}}, \ldots, \frac{z^{m_{l}}}{z^{m_{1}}}, z_{2}, \ldots, z_{m}, z_{m+1}^{ \pm 1}, \ldots, z_{n}^{ \pm 1}\right] \\
& =\operatorname{Spec} K\left[\frac{z_{1}}{z^{m_{1}}}\right] \times \operatorname{Spec} K\left[\frac{z^{m_{2}}}{z^{m_{1}}}, \ldots, \frac{z^{m_{l}}}{z^{m_{1}}}, z_{2}, \ldots, z_{m}, z_{m+1}^{ \pm 1}, \ldots, z_{n}^{ \pm 1}\right] \\
& =\mathbb{A}^{1} \times Y^{\prime}
\end{aligned}
$$

where the second equality follows since $z_{1}$ does not divide $z^{m_{i}}$. Here the strict transform $D$ of $\left\{z_{1}=0\right\}$ is defined by $z_{1} / z^{m_{1}}$, on which $K^{*}$ acts trivially.

It remains to be shown that if we blow up the ideals $I_{c_{i}}^{X}$ for $i \neq 1$ pulled back to $Y$ and normalize, this product structure is preserved. We define the ideals $I_{c_{i}}^{Y}$ on $Y$ generated by all monomials on which $K^{*}$ acts by character $c_{i}$. The lemma below shows that $I_{c_{i}}^{Y}$ is equal to the inverse image of $I_{c_{i}}^{X}$. Hence we may blow up $I_{c_{i}}^{Y}$ instead of the inverse image of $I_{c_{i}}^{X}$. Since $K^{*}$ acts trivially on $z_{1} / z^{m_{1}}$, the ideals $I_{c_{i}}^{Y}$ are generated by monomials in the second term of the product. Thus, blowing up $I_{c_{i}}^{Y}$ preserves the product, and so does normalization.

Lemma 3.2.6. For an affine toric variety $X$ with an action of $K^{*}$ as a oneparameter subgroup of the torus, let $I_{\alpha}^{X}$ be the ideal generated by all monomials on which $K^{*}$ acts by character $\alpha$. If $\phi: Y \rightarrow X$ is a chart of the blowing up of $I_{\alpha}^{X}$, then

$$
I_{\beta}^{Y}=I_{\beta}^{X} \mathcal{O}_{Y}
$$

for all $\beta$.

Proof. Clearly $I_{\beta}^{X} \mathcal{O}_{Y} \subset I_{\beta}^{Y}$. For the converse, let the monomial generators of the coordinate ring of $Y$ be $z_{1} / z_{m_{1}}, z^{m_{2}} / z^{m_{1}}, \ldots, z^{m_{l}} / z^{m_{1}}, z_{1}, \ldots, z_{m}, z_{m+1}^{ \pm 1}, \ldots, z_{n}^{ \pm 1}$ for some generators $z^{m_{i}}$ of $I_{\alpha}$. Thus a regular monomial on $Y$ can be written as a product

$$
z^{m}=\left(\frac{z_{1}}{z^{m_{1}}}\right)^{b_{1}}\left(\frac{z^{m_{2}}}{z^{m_{1}}}\right)^{b_{2}} \cdots\left(\frac{z^{m_{l}}}{z^{m_{1}}}\right)^{b_{l}} \cdot z_{1}^{d_{1}} \cdots z_{n}^{d_{n}}
$$

for some integers $b_{i}, d_{j}$, where $b_{i}, d_{j} \geq 0$ for $i=1, \ldots, l, j=1, \ldots, m$. If $z^{m}$ happens to be a generator of $I_{\beta}^{Y}$, i.e., $K^{*}$ acts on $z^{m}$ by character $\beta$, then also $K^{*}$ acts on $z^{m^{\prime}}=z_{1}^{d_{1}} \cdots z_{n}^{d_{n}}$ by character $\beta$, and $z^{m^{\prime}}$ is in $I_{\beta}^{X} \mathcal{O}_{Y}$.

Corollary 3.2.7. The embeddings $U_{B_{ \pm}^{\text {tor }}} / K^{*} \subset B_{ \pm}^{\text {tor }} / K^{*}$ are toroidal embeddings, and the birational transformation $B_{-}^{\text {tor }} / K^{*} \rightarrow B_{+}^{\text {tor }} / K^{*}$ is toroidal.

Proof. This is immediate from the proposition and Lemma 2.6.1.

In fact, as the following lemma, in conjunction with 3.2.6 shows, the map $B_{-}^{\text {tor }} / K^{*} \rightarrow B_{+}^{\text {tor }} / K^{*}$ is an isomorphism if the set $\left\{c_{1}, \ldots, c_{\mu}\right\}$ in the definition of the torific ideal $I=I_{c_{1}}^{B} \cdots I_{c_{\mu}}^{B}$ is chosen large enough. Since we do not need this result, we only give a sketch of the proof.

Lemma 3.2.8. Let $B=X(N, \sigma)=\operatorname{Spec} K\left[z_{1}, \ldots, z_{m}, z_{m+1}^{ \pm 1}, \ldots, z_{n}^{ \pm 1}\right]$ be a nonsingular affine toric variety, and assume that $K^{*}$ acts on $z_{i}$ by character $c_{i}$. Let $\alpha \in \mathbb{Z}$ be divisible by all $c_{i}$, and let $I_{\alpha}$ and $I_{-\alpha}$ be the ideals generated by all monomials of $K^{*}$-character $\alpha$ and $-\alpha$, respectively. If $\tilde{B}$ is the normalization of the 
blowing up of $I_{\alpha} \cdot I_{-\alpha}$, then the birational map

$$
\tilde{B}_{-} / K^{*} \rightarrow \tilde{B}_{+} / K^{*}
$$

is an isomorphism. The same holds for any torific ideal corresponding to a set of characters containing $\alpha$ and $-\alpha$.

Sketch of the proof. Let $\sigma=\left\langle v_{1}, \ldots, v_{m}\right\rangle$, and let $\pi: N_{\mathbb{R}} \rightarrow N_{\mathbb{R}} / \mathbb{R} \cdot a$ be the projection from $a$. If $\pi$ maps $\sigma$ isomorphically to $\pi(\sigma)$, then $B_{-}$and $B_{+}$are isomorphic already. Otherwise, there exist unique rays $r_{+} \subset \partial_{+} \sigma$ and $r_{-} \subset \partial_{-} \sigma$ such that the star subdivision of $\pi\left(\partial_{+} \sigma\right)$ at $\pi\left(r_{+}\right)$is equal to the star subdivision of $\pi\left(\partial_{-} \sigma\right)$ at $\pi\left(r_{-}\right)$. Now the normalized blowings up of $I_{\alpha}$ and $I_{-\alpha}$ turn out to correspond to star subdivisions of $\sigma$ at $r_{+}$and $r_{-}$. The resulting subdivision $\Sigma$ clearly satisfies $\pi\left(\partial_{-} \Sigma\right)=\pi\left(\partial_{+} \Sigma\right)$.

In our arguments in the next section we will use a more detailed description of the coordinate ring of some affine toric charts of $B^{\text {tor }}$. If $K^{*}$ acts on the variable $z_{i}$ via $t^{c_{i}}$, and if the ideal $I_{c_{i}}$ is not principal, then the strict transform of the divisor $D_{i}=\left\{z_{i}=0\right\}$ is removed from the toroidal structure in $\left(B^{\text {tor }}, D^{\text {tor }}\right)$, i.e., it is not contained in $D^{\text {tor }}$. Assume $\tau$ is a cone in the subdivision associated to the normalization of the blowing up of a torific ideal, and denote the rays in $\tau$ corresponding to the divisors $D_{i}$ which are removed from the toroidal structure by $v_{i}$. After renumbering, we may assume that these are $v_{1}, \ldots, v_{k}$. We have seen above that for each $i=1, \ldots, k$ the corresponding affine toric variety $Y$ decomposes as

$$
Y=\operatorname{Spec} K\left[z_{i} / z^{m_{i}}\right] \times Y_{i}^{\prime} .
$$

Here the strict transform of $D_{i}$ is the zero locus of $z_{i} / z^{m_{i}}$. Since $v_{j} \in \tau$, we have that $\left(v_{i}^{*}-m_{i}, v_{j}\right) \geq 0$ for $i, j=1, \ldots, k$. Since $m_{i}$ is positive on $\tau$, we have

$$
\left(m_{i}, v_{j}\right)=0, \quad i, j=1, \ldots, k,
$$

which means that $z_{j}$ does not divide $z^{m_{i}}$ for $i, j=1, \ldots, k$. We also have that $z_{i}$ does not appear in any monomial in the ring of $Y_{i}^{\prime}$ for $i=1, \ldots, k$.

Applying Lemma 3.2.4 with $w_{i}=v_{i}^{*}-m_{i}$, we obtain the following:

Corollary 3.2.9. Let $B=X(N, \sigma)=\operatorname{Spec} K\left[z_{1}, \ldots, z_{m}, z_{m+1}^{ \pm 1}, \ldots, z_{n}^{ \pm 1}\right]$ be a nonsingular affine toric variety, and assume that $K^{*}$ acts on $z_{i}$ by character $c_{i}$. Let $Y \subset B^{\text {tor }}$ be an affine toric chart corresponding to a cone $\tau$, and assume that the rays in $\tau$ corresponding to divisors which are removed from the toroidal structure are $v_{1}, \ldots, v_{k}$. Then there exist $m_{i} \in \sigma^{\vee}$ and a toric variety $Y^{\prime}$, such that

(1) $\left(m_{i}, v_{j}\right)=0$ for $i, j=1, \ldots, k$,

(2) $z_{i} / z^{m_{i}}$ are $K^{*}$-invariant,

(3) $z_{i}$ does not appear in any monomial in the ring of $Y^{\prime}$, and

$$
Y=\operatorname{Spec} K\left[\frac{z_{1}}{z^{m_{1}}}, \ldots, \frac{z_{k}}{z^{m_{k}}}\right] \times Y^{\prime} .
$$

Example 3.2.10. Consider $B=\mathbb{A}^{3}=\operatorname{Spec} K\left[z_{1}, z_{2}, z_{3}\right]$, where $t \in K^{*}$ acts as

$$
t \cdot\left(z_{1}, z_{2}, z_{3}\right)=\left(t^{2} z_{1}, t^{3} z_{2}, t^{-1} z_{3}\right)
$$


We have the following generators of the torific ideals $I_{\alpha}$ :

$$
\begin{aligned}
I_{2} & =\left(z_{1}, z_{2} z_{3}\right), \\
I_{3} & =\left(z_{2}, z_{1}^{2} z_{3}\right), \\
I_{-1} & =\left(z_{3}\right) .
\end{aligned}
$$

To illustrate Lemma 3.2 .8 we also consider

$$
I_{6}=\left(z_{1}^{3}, z_{2}^{2}, z_{1}^{2} z_{2} z_{3}\right) .
$$

The ideal $I_{-6}=\left(z_{3}^{6}\right)$ is unnecessary here, being principal. Let $I=I_{2} I_{3} I_{6} I_{-1}$. If we regard $B=X(N, \sigma)$ as the toric variety corresponding to the cone

$$
\sigma=\left\langle v_{1}, v_{2}, v_{3}\right\rangle \subset N_{\mathbb{R}}
$$

then $B^{\text {tor }}$ is described by the fan covered by the following four maximal cones:

$$
\begin{aligned}
& \sigma_{1}=\left\langle v_{1}, v_{1}+v_{3}, v_{1}+v_{2}\right\rangle, \\
& \sigma_{2}=\left\langle v_{1}+v_{2}, v_{1}+v_{3}, 2 v_{1}+3 v_{2}, v_{3}\right\rangle, \\
& \sigma_{3}=\left\langle 2 v_{1}+3 v_{2}, v_{3}, 2 v_{2}+v_{1}, v_{1}+v_{2}, v_{2}+v_{3}\right\rangle, \\
& \sigma_{4}=\left\langle 2 v_{2}+v_{1}, v_{2}+v_{3}, v_{2}\right\rangle .
\end{aligned}
$$

If we do not include the factor $I_{6}$ in $I$, then the cones $\sigma_{2}$ and $\sigma_{3}$ are combined to one nonsimplicial cone. Including $I_{6}$ has the effect that $B_{-}^{\text {tor }} / K^{*} \rightarrow B_{+}^{\text {tor }} / K^{*}$ becomes an isomorphism.

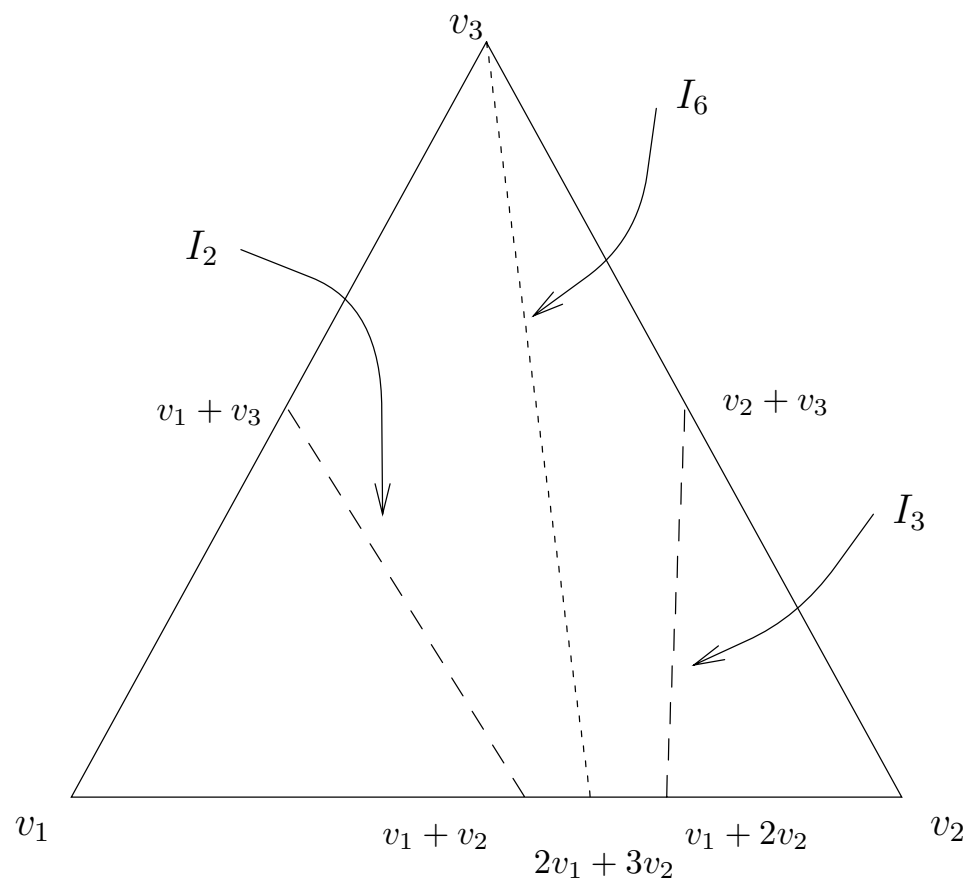

The torifying property can be illustrated on the affine toric variety corresponding to $\sigma_{1}$. The dual cone $\sigma_{1}^{\vee}$ has the product description

$$
\begin{aligned}
\sigma_{1}^{\vee} & =\left\langle v_{1}^{*}-\left(v_{2}^{*}+v_{3}^{*}\right), v_{2}^{*}, v_{3}^{*}\right\rangle \\
& =\left\langle v_{1}^{*}-\left(v_{2}^{*}+v_{3}^{*}\right)\right\rangle \times\left\langle v_{2}^{*}, v_{3}^{*}\right\rangle .
\end{aligned}
$$


Thus, even if we remove the divisor $\left\{z_{1} / z_{2} z_{3}=0\right\}$ from the original toric structure of

$$
X\left(N, \sigma_{1}\right)=\operatorname{Spec} k\left[z_{1} / z_{2} z_{3}, z_{2}, z_{3}\right],
$$

we still have the toroidal embedding structure

$$
X\left(N, \sigma_{1}\right) \backslash\left(\left\{z_{2}=0\right\} \cup\left\{z_{3}=0\right\}\right) \subset X\left(N, \sigma_{1}\right) .
$$

As $z_{1} / z_{2} z_{3}$ is invariant, the action of $K^{*}$ is toroidal. For example, at $0 \in X\left(N, \sigma_{1}\right)$ we have a toric chart

$$
\begin{aligned}
K^{*} \times K^{2} & \rightarrow K \times K^{2} \cong X\left(N, \sigma_{1}\right), \\
\left(x_{1}, x_{2}, x_{3}\right) & \mapsto\left(x_{1}-1, x_{2}, x_{3}\right) .
\end{aligned}
$$

Globally, the divisors corresponding to the new rays

$$
D_{\left\langle v_{1}+v_{2}\right\rangle}, D_{\left\langle v_{1}+v_{3}\right\rangle}, D_{\left\langle 2 v_{1}+3 v_{2}\right\rangle}, D_{\left\langle v_{1}+2 v_{2}\right\rangle}, D_{\left\langle v_{2}+v_{3}\right\rangle}
$$

together with $D_{\left\langle v_{3}\right\rangle}$ coming from $I_{-1}$, are obtained through the blowing up of the torific ideals. Considering

$$
U_{B^{\text {tor }}}=B^{\text {tor }} \backslash\left(D_{\left\langle v_{1}+v_{2}\right\rangle} \cup D_{\left\langle v_{1}+v_{3}\right\rangle} \cup D_{\left\langle 2 v_{1}+3 v_{2}\right\rangle} \cup D_{\left\langle v_{1}+2 v_{2}\right\rangle} \cup D_{\left\langle v_{2}+v_{3}\right\rangle} \cup D_{\left\langle v_{3}\right\rangle}\right)
$$

we obtain a toroidal structure $U_{B^{\text {tor }}} \subset B^{\text {tor }}$ with a toroidal $K^{*}$-action.

\section{A PROOF OF THE WEAK FACTORIZATION THEOREM}

4.1. The situation. In Theorem 2.6 .2 we have constructed a factorization of the given birational map $\phi$ into tightly locally toric birational transformations

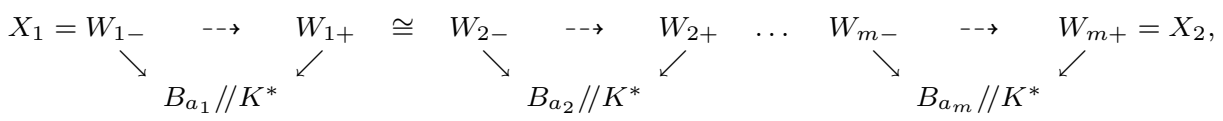

where $W_{i \pm}=\left(B_{a_{i}}\right)_{ \pm} / K^{*}$ (here $W_{i-}$ is $W_{i-1}$ in the notation of Theorem 2.6.2, and $W_{i+}$ is $W_{i}$ ). Since $B$ is nonsingular, we can apply the results of section 3 .

For a choice of a torific ideal $I=I_{c_{1}} \cdots I_{c_{\mu}}$ on $B_{a_{i}}$, denote by $B_{a_{i}}^{\text {tor }} \rightarrow B_{a_{i}}$ the corresponding torific blowing up. Write $W_{i \pm}^{t o r}=B_{a_{i} \pm}^{t o r} / K^{*}$, and $U_{i \pm}^{t o r}=U_{B_{a_{i} \pm}^{t o r}} / K^{*}$. We have a natural diagram of birational maps

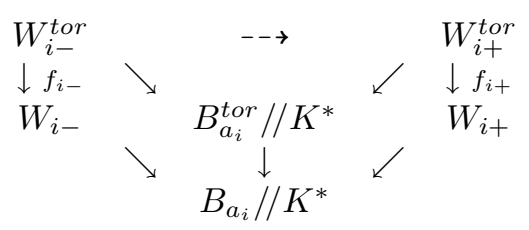

By Corollary 3.2.7 the embeddings $U_{i \pm}^{\text {tor }} \subset W_{i \pm}^{\text {tor }}$ are toroidal, and the birational transformation $\varphi_{i}^{\text {tor }}: W_{i-}^{\text {tor }} \rightarrow W_{i+}^{\text {tor }}$ is toroidal.

We say that the ideal $I=I_{c_{1}} \cdots I_{c_{\mu}}$ is balanced if $\sum c_{j}=0$. It follows from Lemma 3.1.3 that we can always enlarge the set $\left\{c_{1}, \ldots, c_{\mu}\right\}$ to get a balanced torific ideal $I$.

As in section 3 we denote by $\pi: B_{a_{i}} \rightarrow B_{a_{i}} / / K^{*}$ the quotient morphism.

Lemma 4.1.1. Suppose the torific ideal $I$ is balanced. Then the morphism $f_{i \pm}$ is the normalized blowing up of the ideal sheaf $I_{i \pm}$ defined as the pullback to $W_{i \pm}$ of $\pi_{*} I \cap \mathcal{O}_{B_{a_{i}} / / K^{*}}$ 
Proof. By Lemma 3.1.2, the ideal $I$ is generated by $K^{*}$-invariant sections, and we can identify $I$ as the inverse image of an ideal sheaf in $B_{a_{i}} / / K^{*}$ generated by the same sections - which we can take to be $\pi_{*} I \cap \mathcal{O}_{B_{a_{i}} / / K^{*}}$. Let $I_{i \pm}$ be the pullback of this ideal sheaf to $\left(B_{a_{i}}\right)_{ \pm} / K^{*}$ via the map $\left(B_{a_{i}}\right)_{ \pm} / K^{*} \rightarrow B_{a_{i}} / / K^{*}$. Then $f_{i \pm}$ is the normalized blowing up of $I_{i \pm}$ because taking the quotient by $K^{*}$ commutes with blowing up the sheaf $I$.

From now on we assume that the torific ideals are chosen to be balanced. The proof of the main theorem can be carried out without this assumption, but it would make the presentation more complicated.

Note that if the varieties $W_{i \pm}$ were nonsingular and the morphisms $f_{i \pm}$ were composites of blowings up of nonsingular centers, we would get the weak factorization by applying Theorem 1.6.1 to each $\varphi_{i}^{\text {tor }}$. This is not the case in general. In this section we replace $W_{i \pm}$ by nonsingular varieties and $f_{i \pm}$ by composites of blowings up with nonsingular centers.

4.2. Lifting toroidal structures. Let $W_{i \pm}^{r e s} \rightarrow W_{i \pm}$ be the canonical resolution of singularities. Note that, since $W_{i+}=W_{(i+1)-}$, we have $W_{i+}^{\text {res }}=W_{(i+1)-}^{\text {res }}$.

Denote $I_{i \pm}^{r e s}=I_{i \pm} \mathcal{O}_{W_{i \pm}^{r e s}}$. Let $W_{i \pm}^{\text {can }} \rightarrow W_{i \pm}^{r e s}$ be the canonical principalization of the ideal $I_{i \pm}^{r e s}$, and let $h_{i \pm}: W_{i \pm}^{c a n} \rightarrow W_{i \pm}^{\text {tor }}$ be the induced morphism.

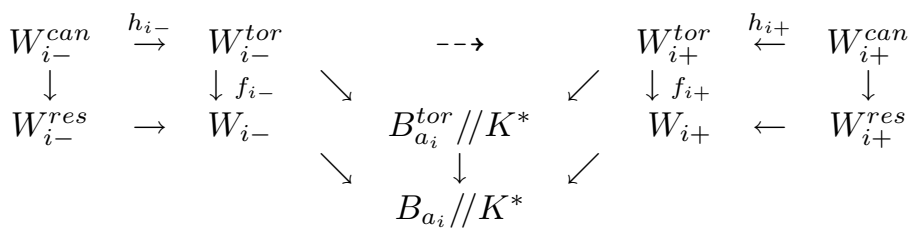

Denote $U_{i \pm}^{\text {can }}=h_{i \pm}^{-1} U_{i \pm}^{\text {tor }}$. The crucial point now is to show:

Proposition 4.2.1. The embedding $U_{i \pm}^{\text {can }} \subset W_{i \pm}^{\text {can }}$ is a toroidal embedding, and the morphism $W_{i \pm}^{\text {can }} \rightarrow W_{i \pm}^{\text {tor }}$ is toroidal.

Proof. For simplicity of notation we drop the subscripts $i$ and $a_{i}$, as we treat each quasi-elementary piece separately. We may assume that all the varieties $B, W_{ \pm}, W_{ \pm}^{\text {tor }}, W_{ \pm}^{\text {res }}, W_{ \pm}^{\text {can }}$ and the morphisms between them are toric. Indeed, if $V_{p} \rightarrow X_{p}$ is a toric chart at some point $p \in W_{ \pm}$, obtained from a toric chart in $B$, we get a toric chart for $W_{ \pm}^{\text {tor }}$ by blowing up a torific ideal in $X_{p}$, which is a toric ideal since it is generated by monomials. Similarly, resolution of singularities and principalization over the toric variety $X_{p}$ provide toric charts for $W_{ \pm}^{\text {res }}$ and $W_{ \pm}^{\text {can }}$. The canonicity of resolution and principalization implies that the maps are toric (i.e., torus equivariant).

Consider now the diagram of toric morphisms between toric varieties and the corresponding diagram of fans:
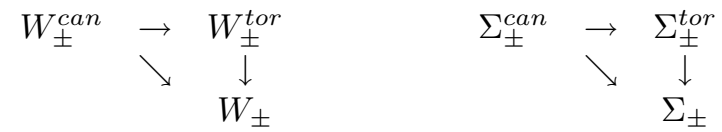

Let $X_{\tau} \subset W_{ \pm}^{\text {tor }}$ be an affine open toric subvariety corresponding to a cone $\tau \in \Sigma_{ \pm}^{\text {tor }}$, and write

$$
X_{\tau} \cong \mathbb{A}^{k} \times X_{\tau^{\prime}}
$$


where the toric divisors $D_{1}, \ldots, D_{k}$ pulled back from $\mathbb{A}^{k}$ are the ones removed in order to define the toroidal structure on $W_{ \pm}^{\text {tor }}$. Let $X_{\tau}^{\text {can }}$ be the inverse image of $X_{\tau}$ in $W_{ \pm}^{c a n}$. We need to show that we have a decomposition $X_{\tau}^{\text {can }} \cong \mathbb{A}^{k} \times X_{\tau^{\prime}}^{\text {can }}$, such that the resulting map $\mathbb{A}^{k} \times X_{\tau^{\prime}}^{\text {can }} \rightarrow \mathbb{A}^{k} \times X_{\tau^{\prime}}$ is a product, with the first factor being the identity map.

Write $X_{\tau}=B_{\tau} / K^{*}$, where $B_{\tau} \subset B_{ \pm}^{\text {tor }}$ is the affine open toric subvariety lying over $X_{\tau}$.

By Corollary 3.2.9 the coordinate rings of $B_{\tau}$ and $X_{\tau}$ can be written as

$$
\begin{aligned}
& A_{X_{\tau}} \cong K\left[\frac{z_{1}}{z^{m_{1}}}, \ldots, \frac{z_{k}}{z^{m_{k}}}\right] \otimes A_{X_{\tau^{\prime}}} \\
& A_{B_{\tau}} \cong K\left[\frac{z_{1}}{z^{m_{1}}}, \ldots, \frac{z_{k}}{z^{m_{k}}}\right] \otimes A_{B_{\tau^{\prime}}},
\end{aligned}
$$

where $X_{\tau^{\prime}}=B_{\tau^{\prime}} / K^{*}$, and where $z^{m_{j}}$ are monomials on which $K^{*}$ acts with the same character as on $z_{j}$, such that $z_{i} \nmid z^{m_{j}}$ for $i, j=1, \ldots, k$.

Lemma 4.2.2. For each $y=\left(y_{1}, \ldots, y_{k}\right) \in K^{k}$ consider the automorphism $\theta_{y}$ of

$$
B=\operatorname{Spec} K\left[z_{1}, \ldots, z_{m}, z_{m+1}^{ \pm 1}, \ldots, z_{n}^{ \pm 1}\right]
$$

defined by

$$
\begin{aligned}
& \theta_{y}\left(z_{i}\right)=z_{i}+y_{i} \cdot z^{m_{i}}, \quad i \leq k, \\
& \theta_{y}\left(z_{i}\right)=z_{i}, \quad i>k \text {. }
\end{aligned}
$$

Then:

(1) $\theta_{y}$ defines an action of the additive group $K^{k}$ on $B$.

(2) The action of $\theta_{y}$ commutes with the given $K^{*}$-action.

(3) The ideals $I_{c}$ are invariant under this action.

(4) The action leaves $B_{ \pm}$invariant, and descends to $W_{ \pm}$.

(5) The action lifts to $B^{\text {tor }}$.

(6) This action on $B^{\text {tor }}$ leaves the open set $B_{\tau}$ invariant.

(7) The induced action on $B_{\tau}$ descends to a fixed-point-free action of $K^{k}$ on $X_{\tau}$.

(8) The resulting action on $X_{\tau}$ is given by

$$
\bar{\theta}_{y}\left(z_{i} / z^{m_{i}}\right)=z_{i} / z^{m_{i}}+y_{i} ; \quad \theta_{y}(f)=f \text { for } f \in A_{X_{\tau^{\prime}}} .
$$

Proof. Since $z_{i} \nmid m_{j}$ for $i, j=1, \ldots, k$, we have that the $\theta_{y}$ commute with each other, and $\theta_{y} \circ \theta_{y^{\prime}}=\theta_{y+y^{\prime}}$ thus defining a $K^{k}$-action. Since $K^{*}$ acts on $z_{i}$ and $m_{i}$ through the same character, it commutes with $\theta_{y}$. For the same reason the ideals $I_{c}$ are invariant: $z^{\alpha}=\prod z_{i}^{\alpha_{i}}$ has $K^{*}$-character $c$ if and only if $\theta_{y}\left(z^{\alpha}\right)$ does, therefore $\theta_{y}^{*} I_{c}=I_{c}$. Since $B_{-}=B \backslash V\left(\sum_{c<0} I_{c}\right)$, we have that $B_{-}$is invariant, and similarly for $B_{+}$; since the $K^{k}$-action commutes with $K^{*}$, it descends to $W_{ \pm}$. Since $I=\prod I_{c_{i}}$, we have that $I$ is $K^{k}$-invariant (i.e., $\theta_{y}^{*} I=I$ ) and therefore the $K^{k}$-action lifts to $B^{\text {tor }}$. Since $z_{j} \nmid m_{i}$ for $i, j=1, \ldots, k$, we have $\theta_{y}\left(z_{i} / z^{m_{i}}\right)=z_{i} / z^{m_{i}}+y_{i}$. Also since $z_{j}$ does not appear in monomials in $A_{B_{\tau^{\prime}}}$, the action on $A_{B_{\tau^{\prime}}}$ is trivial, which implies the rest of the statement.

Back to the proposition. Since $W_{ \pm}^{r e s} \rightarrow W_{ \pm}$is the canonical resolution of singularities, the action of $K^{k}$ lifts to $W_{ \pm}^{\text {res }}$. Since the ideal $I_{ \pm}$is generated by $K^{*}-$ invariants in $I$, and since the action of $K^{*}$ commutes with $\theta_{y}$, we have that $I_{ \pm}$ is invariant under $K^{k}$, and therefore $I_{ \pm}^{r e s}$ is invariant under $K^{k}$ as well. Since $W_{ \pm}^{\text {can }} \rightarrow W_{ \pm}^{\text {res }}$ is the canonical principalization of $I_{ \pm}^{\text {res }}$, the action of $K^{k}$ lifts to $W_{ \pm}^{\text {can }}$. In particular, the map $W_{ \pm}^{\text {can }} \rightarrow W_{ \pm}^{\text {tor }}$ is $K^{k}$-equivariant. By the lemma, the 
action of $K^{k}$ on the invariant open set $X_{\tau} \subset W_{ \pm}^{\text {tor }}$ is fixed-point free, therefore the action on the inverse image $X_{\tau}^{\text {can }}$ is fixed-point free. Writing $X_{\tau^{\prime}}^{\text {can }}$ for the inverse image of the $K^{k}$-slice $(0, \ldots, 0) \times X_{\tau^{\prime}}$, we have that $X_{\tau^{\prime}}^{\text {can }}$ is a $K^{k}$-slice of $X_{\tau}^{\text {can }}$, giving a decomposition $W_{ \pm}^{c a n} \cong \mathbb{A}^{k} \times X_{\tau^{\prime}}^{c a n}$ as needed.

4.3. Conclusion of the proof of Theorem $\mathbf{0 . 1 . 1}$. Since $X_{1}=W_{1-}$ and $X_{2}=$ $W_{m+}$ are nonsingular, we have $W_{1-}^{\text {res }}=W_{1-}$ and $W_{m+}^{r e s}=W_{m+}$. For each $i=$ $1, \ldots, m$ we have obtained a diagram

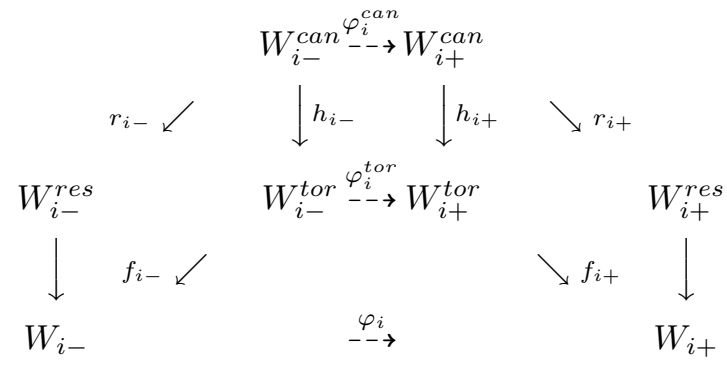

where

(1) the canonical principalizations $r_{i-}$ and $r_{i+}$ are composites of blowings up with nonsingular centers,

(2) $\varphi_{i}^{c a n}$ is a toroidal birational map.

Applying Theorem 2.7.1 to the toroidal map $\varphi_{i}^{c a n}$ we see that $\varphi_{i}^{c a n}$ is a composite of toroidal blowings up and blowings down, with nonsingular centers, between nonsingular toroidal embeddings. Thus we get a factorization

$$
\phi: X_{1}=W_{1-}^{r e s} \rightarrow W_{1+}^{r e s}=W_{2-}^{r e s} \rightarrow \cdots \rightarrow W_{m--\rightarrow}^{r e s} W_{m+}^{r e s}=X_{2},
$$

where all $W_{i}^{\text {res }}$ are nonsingular, and the birational maps are composed of a sequence of blowings up and blowings down. We do not touch the open subset $U \subset X_{1}$ on which $\phi$ is an isomorphism. Projectivity over $X_{2}$ follows from the projectivity statement in Theorem 2.6.2 the projectivity of $W_{ \pm i}^{c a n} \rightarrow W_{ \pm i}$, and the projectivity statement in Theorem [2.7.1] Finally, blowing up a nonsingular center can be factored as a sequence of blowings up of irreducible centers, simply blowing up one connected component at a time; since blowing up is a projective operation, this preserves projectivity. This completes the proof of Theorem 0.1 .1 .

\section{Generalizations}

5.1. Reduction to an algebraically closed overfield. We begin our proof of Theorem 0.3.1. We claim that, in case (1) of algebraic spaces, it suffices to prove the result in case $L$ is algebraically closed. Let $\bar{L}$ be an algebraically closed field containing $L$. Given $\phi: X_{1} \rightarrow X_{2}$, isomorphic on $U$, consider the map $\phi_{\bar{L}}:\left(X_{1}\right)_{\bar{L}} \rightarrow\left(X_{2}\right)_{\bar{L}}$. Assuming the generalized factorization theorem applies over such a field, we get $\varphi_{i \bar{L}}: \bar{V}_{i-1} \rightarrow \bar{V}_{i}$. The functoriality of this factorization guarantees that the Galois group acts on $\bar{V}_{i}$, and $\varphi_{i}$ are Galois equivariant. Therefore, denoting $V_{i}=\bar{V}_{i} / \operatorname{Gal}(\bar{L} / L)$, we get $\varphi_{i}: V_{i-1} \rightarrow V_{i}$ as required. 
5.2. Reduction to an algebraically closed subfield. Still considering case (1), suppose $L \subset K$ are algebraically closed fields, and suppose we have the theorem for algebraic spaces over fields isomorphic to $L$. If $\phi: X_{1} \rightarrow X_{2}$ is a birational map over $L$, with factorization given by $\varphi_{i}: V_{i-1} \rightarrow V_{i}$, then we claim that the induced map $\varphi_{i_{K}}: V_{i-1}{ }_{K} \rightarrow V_{i_{K}}$ is functorial over $K$. Indeed, any isomorphism $K \rightarrow K^{\prime}$ carries $L$ to an isomorphic field, and the functoriality over $L$ induces the desired morphisms $V_{i_{K}} \rightarrow V_{i_{K^{\prime}}^{\prime}}^{\prime}$

5.3. Reduction to $L=\mathbb{C}$. Still considering case (1), let $K$ be algebraically closed and let $\phi: X_{1} \rightarrow X_{2}$ be a birational map of complete algebraic spaces over $K$. Then, by definition, $X_{i}$ are given by étale equivalence relations $R_{i} \subset Y_{i}^{2}$, where $R_{i}$ and $Y_{i}$ are varieties over $K$, and $\phi$ is defined by suitable correspondences between $Y_{i}$. Also the open set $U$ corresponds to a Zariski open set in $Y_{i}$. All these varieties can be defined over a finitely generated subfield $L_{0} \subset K$, and therefore over its algebraic closure $L \subset K$. But any such $L$ can be embedded in $\mathbb{C}$. Therefore, by the previous reductions, it suffices to consider the case of algebraic spaces over a field $L$ isomorphic to $\mathbb{C}$.

By considering the associated analytic spaces, the GAGA principle allows us to use structures (e.g. locally toric, toroidal) defined in the analytic category, as long as we note that the constructions (e.g. birational cobordism, torific ideals) are algebraic, and ensure that the resulting blowings up are functorial in the algebraic sense, namely, independent of a choice of isomorphism $L \rightarrow \mathbb{C}$.

5.4. Reduction to a projective morphism. Now we consider both cases (1) and (2). To simplify the terminology, we use the term "birational map" to indicate also a bimeromorphic map. Given $\phi: X_{1} \rightarrow X_{2}$ isomorphic on $U$, let $X_{i}^{\prime} \rightarrow X_{i}$ be the canonical principalizations of $X_{i} \backslash U$ (endowed with reduced structure). It is convenient to replace $X_{i}$ by $X_{i}^{\prime}$ and assume from now on that $X_{i} \backslash U$ is a simple normal crossings divisor.

We note that Lemma 1.3.1 works word for word in the cases of algebraic spaces or analytic spaces. As we have already remarked, this procedure is functorial. Also, the centers of blowing up have normal crossings with the inverse image of $X_{i} \backslash U$.

It is also easy to see that the resulting morphism $X_{1}^{\prime} \rightarrow X_{2}^{\prime}$ is endowed with a relatively ample line bundle which is functorial under absolute isomorphisms. Indeed, the Proj construction of a blowing up gives a functorial relatively ample line bundle for each blowing up. Furthermore, if $f_{1}: Y_{1} \rightarrow Y_{2}$ and $Y_{2} \rightarrow Y_{3}$ are given relatively ample line bundles $L_{1}$ and $L_{2}$, then there is a minimal positive integer $k$ such that $L_{1} \otimes f_{1}^{*} L_{2}^{\otimes k}$ is relatively ample for $Y_{1} \rightarrow Y_{3}$; thus we can form a functorial relatively ample line bundle for a sequence of blowings up. In an analogous manner we can form a functorial ideal sheaf $I$ on $X_{2}^{\prime}$ such that $X_{1}^{\prime}$ is the blowing up of $I$.

From now on we assume $X_{i} \backslash U$ is a simple normal crossings divisor and $\phi$ is a projective morphism.

5.5. Analytic locally toric structures. There are various settings in which one can generalize locally toric and toroidal structures to algebraic and analytic spaces, either using formal completions (see [42]), or étale charts (see [53]), or logarithmic structures (see [38]). Here we try to keep things simple, by sticking to the analytic situation, and modifying our earlier definitions slightly. 
An analytic toric chart $V_{p} \subset W, \eta_{p}: V_{p} \rightarrow X_{p}$ is defined to be a neighborhood of $p$ in the euclidean topology, with $\eta_{p}$ an open immersion in the euclidean topology. The fact that we use open immersions simplifies our work significantly.

The notions of analytic locally toric structures, analytic toroidal embeddings, modifications, toroidal birational maps and tightly locally toric birational transformations are defined as in the case of varieties, using analytic toric charts.

We note that in an analytic toroidal embedding, the toroidal divisors may have self-intersections. If $U \subset X$ is an analytic toroidal embedding, and if $X^{\prime} \rightarrow X$ is the canonical embedded resolution of singularities of $X \backslash U$, then $X^{\prime} \backslash U$ is a strict toroidal embedding, namely one without self-intersections.

For strict toroidal embeddings, the arguments of 42 regarding rational conical polyhedral complexes, modifications and subdivisions go through, essentially word for word. The divisorial description of the cones (see 42, page 61) shows that the association $(U \subset X) \mapsto \Delta_{X}$ of a polyhedral complex to a toroidal embedding is functorial under absolute isomorphisms in both the analytic and algebraic sense, and similarly for the modification associated to a subdivision.

5.6. Functorial toroidal factorization. Consider an analytic toroidal birational map $\phi: W_{1} \rightarrow W_{2}$ of complete nonsingular toroidal embeddings $U \subset W_{i}$. By the resolution of singularities argument above, we may assume $U \subset W_{i}$ are strict toroidal embeddings. Theorem 2.7.1 applies in this situation, but we need to make the construction functorial. It may be appropriate to rewrite Morelli's proof in a functorial manner, but this would take us beyond the intended scope of this paper. Instead we show here that the result can be made equivariant under the automorphism group of a fan cobordism, which, assuming the axiom of choice, implies functoriality.

Let $\Delta_{i}$ be the polyhedral complex of $U \subset W_{i}$. Denote by $G_{i}$ the automorphism group of $\Delta_{i}$. Since an automorphism of $\Delta_{i}$ is determined by its action on the primitive points of the rays in $\Delta_{i}$, these groups are finite.

Consider the barycentric subdivision $B \Delta_{i} \rightarrow \Delta_{i}$ (see [42, III 2.1, or [5]). It corresponds to a composition of blowings up $B W_{i} \rightarrow W_{i}$, which is functorial. The group $G_{i}$ acts on $B \Delta_{i}$. The subdivision $B \Delta_{i} \rightarrow \Delta_{i}$ has the following property: given a cone $\sigma$ in $B \Delta_{i}$, an element $g \in G_{i}$, and a ray $\tau$ in $\sigma$ such that $g \tau$ is also in $\sigma$, we have $g \tau=\tau$. This means, in particular, that for any subgroup $H \subset G_{i}$ and any $H$-equivariant subdivision $\Delta \rightarrow B \Delta_{i}$ the quotient $\Delta / H$ is also a polyhedral complex (see [5]).

Let $Z$ be the canonical resolution of singularities of the graph of $B W_{1} \rightarrow B W_{2}$. This is clearly functorial in $\phi$. Now $Z \rightarrow B W_{i}$ are toroidal birational morphisms, corresponding to subdivisions $\Delta_{Z} \rightarrow B \Delta_{i}$. Let $H \subset G_{1}$ be the subgroup stabilizing the subdivision $\Delta_{Z} \rightarrow B \Delta_{1}$.

Fix a representative in the isomorphism class of $\Delta_{Z} \rightarrow B \Delta_{1}$, and, using the axiom of choice, fix an isomorphism of any element of the isomorphism class with this representative. Note that the absolute automorphism group of $Z \rightarrow W_{1}$ maps to $H$.

We claim that in order to construct a functorial factorization of $Z \rightarrow W_{1}$ it suffices to construct an $H$-equivariant combinatorial factorization of our representative of the isomorphism class, which by abuse of notation we call $\Delta_{Z} \rightarrow B \Delta_{1}$. Indeed, such a combinatorial $H$-equivariant factorization corresponds to a sequence of $H$ equivariant subdivisions $\Sigma_{i} \rightarrow B \Delta_{1}$ such that either $\Sigma_{i} \rightarrow \Sigma_{i+1}$ or its inverse is a 
nonsingular star subdivision on each cone, such that $\Delta_{Z} \rightarrow B \Delta_{1}$ factors through an isomorphism $\Delta_{Z} \rightarrow \Sigma_{0}$, and such that $\Sigma_{m}=B \Delta_{1}$. Pulling back by the chosen isomorphism, we get an equivariant combinatorial factorization for every element in the isomorphism class, which fit together to give a functorial combinatorial factorization. According to the construction of 42, this functorially corresponds to a sequence of modifications $V_{i} \rightarrow B W_{1}$ which fit together as a functorial factorization of $Z \rightarrow B W_{1}$.

Now $\Delta_{Z} / H \rightarrow B \Delta_{1} / H$ is a subdivision of nonsingular polyhedral complexes, and the toroidal weak factorization theorem says that it admits a combinatorial factorization, as a sequence composed of nonsingular star subdivisions and inverse nonsingular star subdivisions. Lifting these subdivisions to $\Delta_{Z} \rightarrow B \Delta_{1}$, we get an $H$-equivariant factorization, which in turn corresponds to a functorial toroidal factorization of $Z \rightarrow B W_{1}$. We now apply the same procedure to $Z^{\prime} \rightarrow B W_{2}$. This gives the desired functorial toroidal factorization of $\phi$.

5.7. Analytic toroidal $\mathbb{C}^{*}$-actions. The nature of $\mathbb{C}^{*}$-actions on analytic spaces differ significantly from the case of varieties. However, the situation is almost the same if one restricts to relatively algebraic actions.

Definition 5.7.1. Let $X \rightarrow S$ be a morphism of analytic spaces and $L$ a relatively ample line bundle for $X \rightarrow S$. An action of $\mathbb{C}^{*}$ on $X, L$ over $S$ is relatively algebraic if there is an open covering $S=\bigcup S_{i}$, an algebraic action of $\mathbb{C}^{*}$ on a projective space $\mathbb{P}^{N_{i}}$, and a Zariski-locally-closed $\mathbb{C}^{*}$-equivariant embedding $S_{i} \times{ }_{S} X \subset S_{i} \times \mathbb{P}^{N_{i}}$, such that for some integer $l_{i}$ we have that $L_{X \times_{S} S_{i}}^{l_{i}}$ is $\mathbb{C}^{*}$-isomorphic to the pullback of $\mathcal{O}_{\mathbb{P}^{N_{i}}}(1)$.

It is easy to see that if $X \rightarrow S$ is a projective morphism, $L$ a line bundle, with a relatively algebraic $\mathbb{C}^{*}$-action, then $X \subset \mathcal{P} \operatorname{roj}_{S} \operatorname{Sym} E$, where the sheaf $E=\bigoplus_{i=1}^{k} E_{i}$ is a completely reducible $\mathbb{C}^{*}$ sheaf.

In the analytic category we use embedded charts rather than étale ones. Accordingly, we say that a $\mathbb{C}^{*}$-equivariant open set $V \subset X$ is strongly embedded if for any orbit $O \subset V$, the closure of $O$ in $X$ is contained in $V$. This implies that $V / / \mathbb{C}^{*} \rightarrow X / / \mathbb{C}^{*}$ is an open embedding. We define an analytic locally toric $\mathbb{C}^{*}$ action on $W$ using strongly embedded toric charts $\eta_{p}: V_{p} \rightarrow X_{p}$ (we still have the requirement that $V_{p}=\pi^{-1} \pi V_{p}$, where $\pi: W \rightarrow W / / K^{*}$ is the projection, which means that $V_{p} \subset W$ is also strongly embedded).

It is not difficult to show that a strongly embedded toric chart exists for each point $p \in B$, the analogue of Luna's fundamental lemma.

With these modifications, Lemma 1.7 .3 is proven in the same manner in the analytic setting. We also note that, if $D=\sum_{i=1}^{l} D_{i} \subset W$ is a simple normal crossings divisor, then toric charts can be chosen compatible with $D$. Indeed, we only need to choose semi-invariant parameters $x_{1}, \ldots, x_{n}$ so that $x_{i}$ is a defining equation for $D_{i}$, for $i=1, \ldots, l$.

5.8. Analytic birational cobordisms. Analytic birational cobordisms are defined the same way as in the case of varieties, with the extra assumption that the $\mathbb{C}^{*}$-action is relatively algebraic.

Given a projective birational morphism $\phi: X_{1} \rightarrow X_{2}$ we construct a compactified, relatively projective cobordism $\bar{B} \rightarrow X_{2}$ as in the algebraic situation, with the following modification: using canonical resolution of singularities we make the inverse image of $X_{2} \backslash U$ in $\bar{B}$ into a simple normal crossings divisor, crossing $X_{1}$ and 
$X_{2}$ normally. Note that these operations are functorial in absolute isomorphisms of $\phi$.

As indicated before, this construction endows $\bar{B} \rightarrow X_{2}$ with a functorial relatively ample line bundle. Since this bundle is obtained from the Proj construction of the blowing up of an invariant ideal, it comes with a functorial $\mathbb{C}^{*}$-action as well.

The considerations of collapsibility and geometric invariant theory work as in the algebraic setting, leading to Theorem 2.6.2 We note that the resulting locally toric factorization is functorial, and the toric charts on $W_{i}$ can be chosen compatible with the divisor coming from $X_{1} \smile U$ or $X_{2}-U$.

5.9. Functoriality of torification and compatibility with divisors. We note that the definition of the $\alpha$-torific ideals is clearly functorial, and it is easy to make a functorial choice of a balanced set of characters in the construction of a torific ideal (Definition 3.1.4). The proof of its existence works as in the case of varieties. The same is true for its torifying property. In order to make this construction compatible with divisors, we replace the total transform $D$ of $I$ by adding the inverse image of $X_{2} \backslash U$. This guarantees that the resulting toroidal structure on $B^{t o r}$ is compatible with the divisors coming from $X_{2} \backslash U$.

5.10. Conclusion of the proof of Theorem 0.3.1. Canonical resolution of singularities is functorial, therefore the construction of $W_{ \pm}^{\text {res }} \rightarrow W_{ \pm}$is functorial. We can now replace $W_{ \pm}^{\text {res }}$ by the canonical principalization of the inverse image of $X_{2} \backslash U$, making the latter a simple normal crossings divisor. Since the ideal $I$ is functorial, the construction of $W_{ \pm}^{\text {can }} \rightarrow W_{ \pm}$is functorial, and the locally toric structure implies that the centers of blowing up in $W_{ \pm}^{\text {can }} \rightarrow W_{ \pm}^{\text {res }}$ have normal crossings with the inverse image of $X_{2}-U$. We can now apply functorial toroidal factorization to the toroidal birational map $W_{-}^{\text {can }} \rightarrow W_{+}^{\text {can }}$. Note that the centers of blowing up, being toroidal, automatically have normal crossings with $W_{ \pm}^{\text {can }} \backslash U_{ \pm}^{\text {can }}$. The theorem follows.

\section{Problems Related to Weak factorization}

6.1. Strong factorization. Despite our attempts, we have not been able to use the methods of this paper to prove the strong factorization conjecture, even assuming the toroidal case holds true.

In the construction of the torific ideal in 3.1 and the analysis of its blowing up in 3.2 and 4.2, the assumption of the cobordism $B_{a_{i}}$ being quasi-elementary is essential. It is easy to give examples where the formation of the torific ideal does not commute with taking affine open sets, therefore we cannot glue together the ideals defined on the individual quasi-elementary pieces into one ideal over the entire birational cobordism $B$.

One can extend each of these ideals separately, for instance by taking the Zariski closure of its zero scheme, but the behavior of this extension (as well as others we have considered) along $B-B_{a_{i}}$ is problematic.

The weak factorization theorem reduces the strong factorization conjecture to the following problem:

Problem 6.1.1. Let $X_{1} \rightarrow X_{2} \rightarrow \cdots \rightarrow X_{n}$ be a sequence of blowings up with nonsingular centers, with $X_{n}$ nonsingular, and such that the center of blowing up of $X_{i} \rightarrow X_{i+1}$ has normal crossings with the exceptional divisor of $X_{i+1} \rightarrow X_{n}$. 
Let $Y \rightarrow X_{n}$ be a blowing up with nonsingular center. Find a strong factorization of the birational map $X_{1} \rightarrow Y$.

We believe that at least the threefold case of this problem is tractable.

\subsection{Toroidalization.}

Problem 6.2.1 (Toroidalization). Let $\phi: X \rightarrow Y$ be a surjective proper morphism between complete nonsingular varieties over an algebraically closed field of characteristic 0. Do there exist sequences of blowings up with nonsingular centers $\nu_{X}: \tilde{X} \rightarrow X$ and $\nu_{Y}: \tilde{Y} \rightarrow Y$ so that the induced map $\tilde{\phi}: \tilde{X} \rightarrow \tilde{Y}$ is a toroidal morphism? Can such maps be chosen in a functorial manner, and in such a way that they preserve any open set where $\phi$ admits a toroidal structure?

This can be viewed as a problem of finding a Hironaka-type logarithmic desingularization of a morphism. The result of [3], Theorem 2.1, gives a logarithmic desingularization of a morphism, but not using blowings up with nonsingular centers.

A similar conjecture was proposed in [43. We note that the toroidalization conjecture concerns not only birational morphisms $\phi$ but also generically finite morphisms or morphisms with $\operatorname{dim} X>\operatorname{dim} Y$. The solution to the above conjecture would reduce the strong factorization conjecture to the toroidal case, simply by considering the case of a birational morphism $\phi$ and then applying the toroidal case to $\tilde{\phi}$. Until recently the authors knew of a complete proof only if either $\operatorname{dim} X=2$ (see below), or $\operatorname{dim} Y=1$ (which follows immediately from resolution of singularities; see [42], II §3). Recently, S. D. Cutkosky worked out a highly nontrivial solution of the case $\operatorname{dim} X=3, \operatorname{dim} Y=2[18]$.

The conjecture is false in positive characteristics due to wild ramifications. See, e.g., [19].

One general result which we do know is the following.

Theorem 6.2.2. Let $\phi: X \rightarrow Y$ be a surjective morphism between complete varieties over an algebraically closed field of characteristic 0 . Then there exists a modification $\nu_{X}: \tilde{X} \rightarrow X$ and a sequence of blowings up with nonsingular centers $\nu_{Y}: \tilde{Y} \rightarrow Y$ so that the induced map $\tilde{\phi}: \tilde{X} \rightarrow \tilde{Y}$ is a toroidal morphism.

Proof. In 3], Theorem 2.1, it is shown that modifications $\nu_{X}$ and $\nu_{Y}$ such that $\tilde{\phi}$ is toroidal exist, assuming $X$ and $Y$ are projective and the generic fiber of $\phi$ is geometrically integral. We can reduce to the projective case using Chow's lemma. The case where the generic fiber is not geometrically integral is resolved in the second author's thesis [37. Since the latter is not widely available we give a similar argument here. The inductive proof of [3], Theorem 2.1, reduces the problem to the case where $\phi$ is generically finite. By Hironaka's flattening (or by taking a resolution of the graph of $Y \rightarrow H_{i l b_{Y}}(X)$ ), we may assume that $X \rightarrow Y$ is finite. Using resolution of singularities, we may assume $Y$ is nonsingular and the branch locus is a normal crossings divisor. By normalizing $X$ we may assume $X$ normal. Denoting the complement of the branch locus by $U_{Y}$ and its inverse image in $X$ by $U_{X}$, Abhyankar's lemma says that $U_{X} \subset X$ is a toroidal embedding and $X \rightarrow Y$ is toroidal, which is what we needed.

It remains to be shown that $\nu_{Y}$ can be chosen to be a sequence of blowings up with nonsingular centers. Let $Y \leftarrow Y^{\prime} \rightarrow \tilde{Y}$ be an elimination of indeterminacies of $Y \rightarrow \tilde{Y}$ and let $Y^{\prime \prime} \rightarrow Y^{\prime}$ be the canonical principalization of the pullback of the 
ideal of the toroidal divisor of $\tilde{Y}$. Let $X^{\prime \prime} \rightarrow Y^{\prime \prime} \times_{\tilde{Y}} \tilde{X}$ be the normalization of the dominant component. Then $Y^{\prime \prime} \rightarrow Y$ is a sequence of blowings up with nonsingular centers. Applying [3, Lemma 6.2, we see that $X^{\prime \prime} \rightarrow Y^{\prime \prime}$ is still toroidal, which is what we needed.

Since every proper birational morphism of nonsingular surfaces factors as a sequence of point blowings up, we get:

Corollary 6.2.3. The toroidalization conjecture holds for a generically finite morphism $\phi: X \rightarrow Y$ of surfaces.

In this case, it is not difficult to deduce that there exists a minimal toroidalization (since the configuration of intermediate blowings up in $\tilde{X} \rightarrow X$ or $\tilde{Y} \rightarrow Y$ forms a tree). This result has been proven in an algorithmic manner by Cutkosky and Piltant [19]. Similar statements can be found in [6].

\section{ACKNOWLEDGEMENTS}

We heartily thank E. Bierstone, L. Bonavero, S. Iitaka, Y. Kawamata, P. Milman, Y. Miyaoka, S. Mori, N. Nakayama B. Siebert, and V. Srinivas for helpful comments.

\section{REFERENCES}

[1] S. Abhyankar, On the valuations centered in a local domain, Amer. J. Math. 78 (1956), 321-348. MR 18:556b

[2] D. Abramovich and A. J. de Jong, Smoothness, semistability, and toroidal geometry, J. Alg. Geom. 6 (1997), 789-801. MR 99b:14016

[3] D. Abramovich and K. Karu, Weak semistable reduction in characteristic 0, Invent. Math. 139 (2000), no. 2, 241-273. MR 2001f:14021

[4] D. Abramovich, K. Matsuki and S. Rashid, A note on the factorization theorem of toric birational maps after Morelli and its toroidal extension, Tohoku Math. J. (2) 51 (1999), no. 4, 489-537. MR 2000i:14073 Correction: Tohoku Math. J. 52 (2000), 629-631. MR 2001j:14069

[5] D. Abramovich and J. Wang, Equivariant resolution of singularities in characteristic 0, Math. Res. Letters 4 (1997), 427-433. MR 98c:14011

[6] S. Akbulut and H. King, Topology of algebraic sets, MSRI publications 25. MR 94m:57001

[7] V. V. Batyrev, Stringy Hodge numbers of varieties with Gorenstein canonical singularities, in Integrable systems and algebraic geometry (Kobe/Kyoto, 1997), 1-32, World Sci. Publishing, Rivers Edge, NJ, 1998. MR 2001a:14039

[8] V. V. Batyrev, Non-Archimedean integrals and stringy Euler numbers of log-terminal pairs, J. Eur. Math. Soc. 1 (1999), no. 1, 5-33. MR 2001j:14018

[9] E. Bierstone and D. Milman, Canonical desingularization in characteristic zero by blowing up the maximum strata of a local invariant, Invent. Math. 128 (1997), 207-302. MR 98e: 14010

[10] F. Bittner, The universal Euler characteristic for varieties of characteristic zero, preprint math.AG/0111062.

[11] L. A. Borisov and A. Libgober, Elliptic Genera of singular varieties, preprint math. AG/0007108.

[12] M. Brion and C. Procesi, Action d'un tore dans une variété projective, in Operator algebras, unitary representations, enveloping algebras, and invariant theory (Paris, 1989), 509-539, Progr. Math., 92, Birkhäuser, Boston, MA, 1990. MR 92m:14061

[13] C. Christensen, Strong domination/weak factorization of three dimensional regular local rings, Journal of the Indian Math. Soc. 45 (1981), 21-47. MR 88a:14010b

[14] A. Corti, Factoring birational maps of 3-folds after Sarkisov, J. Alg. Geom. 4 (1995), 23-254. MR 96c:14013

[15] B. Crauder, Birational morphisms of smooth algebraic threefolds collapsing three surfaces to a point, Duke Math. J. 48 (1981), 589-632. MR 83a:14012 
[16] S. D. Cutkosky, Local factorization of birational maps, Advances in Math. 132 (1997), 167-315. MR 99c:14018

[17] S. D. Cutkosky, Local monomialization and factorization of morphisms, Astérisque 260, Soc. Math. France, 1999. MR 2001c:14027

[18] S. D. Cutkosky, Monomialization of morphisms from 3-folds to surfaces, preprint math.AG/0010002

[19] S. D. Cutkosky and O. Piltant, Monomial resolutions of morphisms of algebraic surfaces, Special issue in honor of Robin Hartshorne. Comm. Algebra 28 (2000), no. 12, 5935-5959. MR 2002a:14012

[20] V. I. Danilov, The geometry of toric varieties, Russian Math. Surveys 33 (1978), no. 2, 97-154. MR 80g:14001

[21] V. I. Danilov, Birational geometry of toric 3-folds, Math. USSR-Izv. 21 (1983), 269-280. MR 84e:14008

[22] J. Denef and F. Loeser, Germs of arcs on singular algebraic varieties and motivic integration. Invent. Math. 135 (1999), no. 1, 201-232. MR 99k:14002

[23] I. V. Dolgachev and Y. Hu, Variation of geometric invariant theory quotients, Inst. Hautes Études Sci. Publ. Math. No. 87 (1998), 5-56. MR 2000b:14060

[24] G. Ewald, Blow-ups of smooth toric 3-varieties, Abh. Math. Sem. Univ. Hamburg 57 (1987), 193-201. MR 89b:14065

[25] J. Franke, Riemann-Roch in functorial form, preprint 1992, $78 \mathrm{pp}$

[26] W. Fulton, Introduction to toric varieties, Annals of Mathematics Studies 131, Princeton University Press, 1993. MR 94g:14028

[27] H. Gillet and Ch. Soulé, Direct images in non-archimedean Arakelov theory, Ann. Inst. Fourier (Grenoble) 50 (2000), no. 2, 363-399. MR 2001j:14036

[28] H. Hironaka, On the theory of birational blowing-up, Harvard University Ph.D. Thesis 1960.

[29] H. Hironaka, An example of a non-Kälerian complex-analytic deformation of Kählerian complex structure, Annals of Math. (2) 75 (1962), 190-208. MR 25:2618

[30] H. Hironaka, Resolution of singularities of an algebraic variety over a field of characteristic zero, Annals of Math. 79 (1964), 109-326. MR 33:7333

[31] H. Hironaka, Flattening theorem in complex analytic geometry, Amer. J. of Math. 97 (1975), no. 2, 503-547. MR 52:14365

[32] Y. Hu, The geometry and topology of quotient varieties of torus actions, Duke Math. J. 68 (1992), no. 1, 151-184; Erratum: Duke Math. J. 68 (1992), no. 3, 609. MR 93k:14019a

[33] Y. Hu, Relative geometric invariant theory and universal moduli spaces, Internat. J. Math. 7 (1996), no. 2, 151-181. MR 98i:14016

[34] Y. Hu and Ś. Keel, A GIT proof of Wtodarczyk's weighted factorization theorem, preprint math. AG/9904146.

[35] S. Iitaka, Algebraic Geometry (An Introduction to Birational geometry of Algebraic Varieties), Graduate Texts in Math. 76, 1982. MR 84j:14001

[36] A. J. de Jong, Smoothness, semistability, and alterations, Publ. Math. I.H.E.S. 83 (1996), 51-93. MR 98e:14011

[37] K. Karu, Boston University dissertation, 1999. http://math.bu.edu/people/kllkr/th.ps

[38] K. Kato, Toric singularities, Amer. J. Math. 116 (1994), 1073-1099. MR 95g:14056]

[39] Y. Kawamata, On the finiteness of generators of a pluricanonical ring for a 3-fold of general type, Amer. J. Math. 106 (1984), no. 6, 1503-1512. MR 86j:14032

[40] Y. Kawamata, The cone of curves of algebraic varieties, Ann. of Math. (2) 119 (1984), no. 3, 603-633. MR 86c:14013b

[41] Y. Kawamata, Crepant blowing-ups of three dimensional canonical singularities and its application to degenerations of surfaces, Ann. of Math. 127 (1988), 93-163. MR 89d:14023

[42] G. Kempf, F. Knudsen, D. Mumford and B. Saint-Donat, Toroidal embeddings I, 339, Lecture Notes in Mathematics, Springer, 1973. MR 49:299

[43] H. King, Resolving Singularities of Maps, Real algebraic geometry and topology (East Lansing, Michigan 1993), Contemp. Math., Amer. Math. Soc., 1995. MR 96e:14016

[44] J. Kollár, The cone theorem. Note to a paper: "The cone of curves of algebraic varieties" ([40]) by Y. Kawamata, Ann. of Math. (2) 120 (1984), no. 1, 1-5. MR 86c:14013c

[45] M. Kontsevich, Lecture at Orsay (December 7, 1995).

[46] V. S. Kulikov, Decomposition of a birational map of three-dimensional varieties outside codimension 2, Math. USSR Izvestiya 21 (1983), 187-200. MR 84j:14022 
[47] G. Lachaud and M. Perret, Un invariant birationnel des variétés de dimension 3 sur un corps fini J. Algebraic Geom. 9 (2000), no. 3, 451-458. MR 2001g:14036

[48] M. N. Levine and F. Morel, Cobordisme Algébrique II, C. R. Acad. Sci. Paris 332 (2001), no. $9,815-820$.

[49] M. N. Levine and F. Morel, Algebraic cobordism, preprint.

[50] E. Looijenga, Motivic measures, preprint math.AG/0006220

[51] D. Luna, Slices étales. Sur les groupes algébriques, pp. 81-105. Bull. Soc. Math. France, Paris, Memoire 33. Soc. Math. France, Paris, 1973. MR 49:7269

[52] K. Matsuki, Introduction to the Mori program, Universitext, Springer Verlag, Berlin, 2001.

[53] K. Matsuki, Lectures on factorization of birational maps, RIMS preprint, 1999.

[54] J. Milnor, Morse Theory, Annals of Math. Stud. 51, Princeton Univ. Press, 1963. MR 29:634

[55] B. Moishezon, On $n$-dimensional compact varieties with $n$ algebraically independent meromorphic functions, Amer. Math. Soc. Transl. 63 (1967), 51-177. MR 35:7355a,b,c

[56] R. Morelli, The birational geometry of toric varieties, J. Alg. Geom. 5 (1996), 751-782. MR 99b:14056

[57] R. Morelli, Correction to "The birational geometry of toric varieties", 1997 http://www.math.utah.edu/ morelli/Math/math.html

[58] S. Mori, Threefolds whose canonical bundles are not numerically effective, Annals of Math. 116 (1982), 133-176. MR 84e:14032

[59] S. Mori, Flip theorem and the existence of minimal models for 3-folds, Journal of AMS 1 (1988), 117-253. MR 89a:14048

[60] D. Mumford, J. Fogarty and F. Kirwan, Geometric Invariant Theory (Third Enlarged Edition), Ergebnisse der Mathematik und ihrer Grenzgebiete, 34, Springer-Verlag, 1992. MR 95m:14012

[61] T. Oda, Torus embeddings and applications, Based on joint work with Katsuya Miyake. Tata Inst. Fund. Res., Bombay, 1978. MR 81e:14001

[62] T. Oda, Convex Bodies and Algebraic Geometry, Springer-Verlag, 15, 1988. MR 88m:14038

[63] R. Pandharipande, A compactification over $\bar{M}_{g}$ of the universal moduli space of slopesemistable vector bundles, J. Amer. Math. Soc. 9 (1996), no. 2, 425-471. MR 96f:14014

[64] H. Pinkham, Factorization of birational maps in dimension 3, Proceedings of Symposia in Pure Math. 40, 1983. MR 85g:14015

[65] M. Raynaud and L. Gruson, Critères de platitude et de projectivité. Techniques de "platification" d'un module, Invent. Math. 13 (1971), 1-89. MR 46:7219

[66] M. Reid, Canonical threeefolds, Géométrie Algémétrie Algébrique Angers 1979, A. Beauville, ed., Sijthoff and Nordhoff, 1980, p. 273-310. MR 82i:14025

[67] M. Reid, Minimal models of canonical 3-folds, Adv. Stud. in Pure Math. 1 (1983), 131-180. MR 86a:14010

[68] M. Reid, Decomposition of Toric Morphisms, Arithmetic and Geometry, papers dedicated to I. R. Shafarevich on the occasion of his 60th birthday, vol. II, Progress in Math. (M. Artin and J. Tate, eds.), 36, 1983, pp. 395-418. MR 85e:14071

[69] M. Reid, Birational geometry of 3-folds according to Sarkisov, preprint 1991.

[70] J. Sally, Regular overrings of regular local rings, Trans. Amer. Math. Soc. 171 (1972), 291300. MR 46:9033 Erratum: Trans. Amer. Math. Soc. 213 (1975), 429. MR 52:3143

[71] V. G. Sarkisov, Birational maps of standard $\mathbb{Q}$-Fano fiberings, I. V. Kurchatov Institute Atomic Energy preprint, 1989.

[72] M. Schaps, Birational morphisms of smooth threefolds collapsing three surfaces to a curve, Duke Math. J. 48 (1981), 401-420. MR 83h:14012

[73] D. L. Shannon, Monoidal transforms of regular local rings, Amer. J. Math. 45 (1973), 284320. MR 48:8492

[74] V. V. Shokurov, A nonvanishing theorem, Izv. Akad. Nauk SSSR Ser. Mat. 49 (1985), no. 3, 635-651. MR 87j:14016

[75] H. Sumihiro, Equivariant Completion I, II, J. Math. Kyoto Univ. 14, 15 (1974), (1975), 1-28, 573-605. MR 49:2732 MR 52:8137

[76] M. Teicher, Factorization of a birational morphism between 4-folds. Math. Ann. 256 (1981), no. 3, 391-399. MR 82k:14014

[77] M. Thaddeus, Stable pairs, linear systems and the Verlinde formula, Invent. Math. 117 (1994), 317-353. MR 95e:14006 
[78] M. Thaddeus, Geometric invariant theory and flips, J. Amer. Math. Soc. 9 (1996), 691-723. MR 96m:14017

[79] O. Villamayor, Constructiveness of Hironaka's resolution. Ann. Sci. École Norm. Sup. (4) 22 (1989), no. 1, 1-32. MR 90b:14014

[80] J. Włodarczyk, Decomposition of Birational Toric Maps in Blow-Ups and Blow-Downs. A Proof of the Weak Oda Conjecture, Transactions of the AMS 349 (1997), 373-411. MR 97d:14021

[81] J. Włodarczyk, Birational cobordism and factorization of birational maps, J. Alg. Geom. 9 (2000), no. 3, 425-449. MR 2002d:14019

[82] J. Włodarczyk, Toroidal Varieties and the Weak Factorization Theorem, preprint math.AG/9904076.

[83] O. Zariski, Algebraic Surfaces, Springer-Verlag, 1934. MR 57:9695

Department of Mathematics, Boston University, 111 Cummington Street, Boston, MASSAChUSETTS 02215

E-mail address: abrmovic@math.bu.edu

Department of Mathematics, Harvard University, One Oxford Street, Cambridge, Massachusetts 02139

E-mail address: kkaru@math.harvard.edu

Department of Mathematics, Purdue University, 1395 Mathematical Sciences BuildING, West Lafayette, Indiana 47907-1395

E-mail address: kmatsuki@math.purdue.edu

Instytut Matematyki UW, Banacha 2, 02-097 Warszawa, Poland

E-mail address: jwlodar@mimuw.edu.pl 